Homology, Homotopy and Applications, vol.16(2), 2014, pp.345-369

\title{
HOMOTOPY REPRESENTATIONS OVER THE ORBIT CATEGORY
}

\author{
IAN HAMBLETON AND ERGÜN YALÇIN
}

(communicated by J. Daniel Christensen)

\begin{abstract}
Let $G$ be a finite group. The unit sphere in a finitedimensional orthogonal $G$-representation motivates the definition of homotopy representations, due to tom Dieck. We introduce an algebraic analogue and establish its basic properties, including the Borel-Smith conditions and realization by finite $G$-CW-complexes.
\end{abstract}

\section{Introduction}

Let $G$ be a finite group. The unit spheres $S(V)$ in finite-dimensional orthogonal representations of $G$ provide the basic examples of smooth $G$-actions on spheres. Moreover, character theory reveals intricate relations between the dimensions of the fixed subspheres $S(V)^{H}$, for subgroups $H \leqslant G$, and the structure of the isotopy subgroups $\left\{G_{x} \mid x \in S(V)\right\}$. Our goal is to better understand the constraints on these basic invariants, in order to construct new smooth non-linear finite group actions on spheres $($ see $[\mathbf{8}, \mathbf{9}])$.

In order to put this problem in a more general setting, tom Dieck [12, II.10.1] introduced geometric homotopy representations, as finite $G$-CW-complexes $X$ with the property that each fixed set $X^{H}$ is homotopy equivalent to a sphere. In this paper, we study an algebraic version of this notion for $R$-module chain complexes over the orbit category $\Gamma_{G}=\operatorname{Or}_{\mathcal{F}} G$, with respect to a ring $R$ and a family $\mathcal{F}$ of subgroups of $G$. We usually work with $R=\mathbb{Z}_{(p)}$, for some prime $p$, or $R=\mathbb{Z}$. This theory was developed by Lück $[\mathbf{1 0}, \S 9, \S 17]$ and tom Dieck [12, §10-11].

The homological dimensions of the various fixed sets are encoded in a conjugationinvariant function $\underline{n}: \mathcal{S}(G) \rightarrow \mathbb{Z}$, where $\mathcal{S}(G)$ denotes the set of subgroups of $G$. The function $\underline{n}$ is supported on the family $\mathcal{F}$, if $\underline{n}(H)=-1$ for $H \notin \mathcal{F}$ (see Definition 2.4). We say that a finite projective chain complex $\mathbf{C}$ over $R \Gamma_{G}$ is an $R$-homology $\underline{n}$-sphere if the reduced homology of $\mathbf{C}(K)$ is the same as the reduced homology of an $\underline{n}(K)$ sphere (with coefficients in $R$ ) for all $K \in \mathcal{F}$.

If $\mathbf{C}$ is an $R$-homology $\underline{n}$-sphere, which satisfies the internal homological conditions observed for representation spheres (see Definition 2.8), then we say that $\mathbf{C}$ is an

Research partially supported by NSERC Discovery Grant A4000. The second author is partially supported by TÜBİTAK-TBAG/110T712.

Received February 13, 2014, revised August 1, 2014; published on November 24, 2014.

2010 Mathematics Subject Classification: 57S17, 55U15, 20J05, 18 G35.

Key words and phrases: homotopy representation, orbit category.

Article available at http://dx.doi.org/10.4310/HHA.2014.v16.n2.a19

Copyright (C) 2014, International Press. Permission to copy for private use granted. 
algebraic homotopy representation. By [12, II.10], these conditions are all necessary for $\mathbf{C}$ to be chain homotopy equivalent to a geometric homotopy representation. In Proposition 2.10, we show more generally that these conditions hold for $\mathbf{C}$ an $R$ homology $\underline{n}$-sphere, whenever $\underline{n}=\operatorname{Dim} \mathbf{C}$, where $\operatorname{Dim} \mathbf{C}$ denotes the chain dimension function of $\mathbf{C}$. When this equality holds, we say that $\mathbf{C}$ is a tight complex.

In general, $\underline{n}(K) \leqslant \operatorname{Dim} \mathbf{C}(K)$ for each $K \in \mathcal{F}$, and one would expect obstructions to finding a tight complex which is chain homotopy equivalent to a given $R$-homology $\underline{n}$-sphere. Our first main result shows the relevance of the internal homological conditions for this question.

Theorem A. Let $G$ be a finite group, $\mathcal{F}$ be a family of subgroups of $G$, and $R$ be a principal ideal domain. Suppose that

(i) $\underline{n}: \mathcal{S}(G) \rightarrow \mathbb{Z}$ is a conjugation-invariant function supported on $\mathcal{F}$, and

(ii) $\mathbf{C}$ is a finite chain complex of free $R \Gamma_{G}$-modules that is an $R$-homology $\underline{n}$-sphere.

Then $\mathbf{C}$ is chain homotopy equivalent to a finite free chain complex $\mathbf{D}$ satisfying $\underline{n}=\operatorname{Dim} \mathbf{D}$ if and only if $\mathbf{C}$ is an algebraic homotopy representation.

Theorem A was motivated by [8, Theorem 8.10], which states that a finite chain complex of free $\mathbb{Z} \Gamma_{G}$-modules can be realized by a geometric $G$-CW-complex if it is a tight homology $\underline{n}$-sphere such that $\underline{n}(H) \geqslant 3$ for all $H \in \mathcal{F}$. Upon combining these two statements, we get the following geometric realization result.

Corollary B. Let $\mathbf{C}$ be a finite chain complex of free $\mathbb{Z} \Gamma_{G}$-modules which is a homology $\underline{n}$-sphere. If $\mathbf{C}$ is an algebraic homotopy representation, and in addition, if $\underline{n}(K) \geqslant 3$ for all $K \in \mathcal{F}$, then there is a finite $G$-CW-complex $X$, with isotropy in $\mathcal{F}$, such that $\mathbf{C}\left(X^{?} ; \mathbb{Z}\right)$ is chain homotopy equivalent to $\mathbf{C}$ as chain complexes of $\mathbb{Z} \Gamma_{G}$-modules.

We are interested in constructing finite $G-\mathrm{CW}$-complexes with some restrictions on the family of isotropy subgroups. We say a $G$-CW-complex $X$ has rank 1 isotropy if for every $x \in X$, the isotropy subgroup $G_{x}$ has rank $G_{x} \leqslant 1$. Recall that rank of a finite group $G$ is defined as the largest integer $k$ such that $(\mathbb{Z} / p)^{k} \leqslant G$ for some prime $p$. We will use Theorem A and Corollary B to study the following:

Question. Which finite groups $G$ admit a finite $G$-CW-complex $X$ with rank 1 isotropy, such that $X$ is homotopy equivalent to a sphere?

One motivation for this work is that rank 1 isotropy examples lead to free $G$-CWcomplex actions of finite groups on products of spheres (see Adem and Smith [1]).

In $[\mathbf{8}]$ we gave the first non-trivial example, by constructing a finite $G$-CW-complex $X \simeq S^{n}$ for the symmetric group $G=S_{5}$, with cyclic 2-group isotropy. However, the arguments used special features of the isotropy family. Corollary B now provides an effective general method for the geometric realization of algebraic models. The algebraic homotopy representation conditions are easy to check locally over $R=\mathbb{Z}_{(p)}$ at each prime, and fit well with the local-to-global procedure for constructing chain complexes $\mathbf{C}$ over $\mathbb{Z} \Gamma_{G}$. In a sequel $[\mathbf{9}]$ to this paper, we apply Corollary B to construct infinitely many new examples with rank 1 isotropy, for certain interesting families of rank 2 groups. 
In Section 5 we consider the algebraic version of a well-known theorem in transformation groups: the dimension function of a homotopy representation satisfies certain conditions called the Borel-Smith conditions (see Definition 5.1).

Theorem C. Let $G$ be a finite group, $R=\mathbb{Z} / p$, and $\mathcal{F}$ be a given family of subgroups of $G$. If $\mathbf{C}$ is a finite projective chain complex over $R \Gamma_{G}$, which is an R-homology $\underline{n}$-sphere, then the function $\underline{n}$ satisfies the Borel-Smith conditions at the prime $p$.

A similar result was mentioned by Grodal and Smith in [7]. As an application of Theorem C, we show that such a finite projective chain complex over $R \Gamma_{G}$ does not exist for the group $G=\mathrm{Qd}(p)$ with respect to the family $\mathcal{F}$ of rank 1 subgroups (see Example 5.13 and Proposition 5.14). This is an important group theoretic constraint on the existence question for geometric homotopy representations with rank 1 isotropy (see Ünlü [13]).

One of the main ideas in the proof of Theorem $\mathrm{C}$ is the reduction of a given chain complex of $R \Gamma_{G}$-module $\mathbf{C}$ to a chain complex over $R \Gamma_{K / N}$ for a subquotient $K / N$ appearing in the Borel-Smith conditions. For this reduction, we introduce inflation and deflation of modules over the orbit category, via restriction and induction associated to a certain functor $F$ (see Section 4 ). Then we use spectral sequence arguments to conclude that the conditions given in the Borel-Smith conditions hold for these reduced chain complexes over $R \Gamma_{K / N}$.

Here is a brief outline of the paper. In Section 2 we give the precise setting and background definitions for the concepts just presented (see Definition 2.8) and prove the "only if" direction of Theorem A. The "if" direction of Theorem A is proved in Section 3, together with Corollary B. In Section 5 we discuss the Borel-Smith conditions and prove Theorem C.

Our methods involve the study of finite-dimensional chain complexes of finitely generated projective modules over the orbit category, called finite projective chain complexes, for short. Such chain complexes are the algebraic analogue of finitelydominated $G$-CW complexes.

\section{Acknowledgments}

We would like to thank Alejandro Adem, Jesper Grodal, and Jeff Smith for helpful conversations on various occasions. We also thank the referees for their many constructive comments and suggestions.

\section{Algebraic homotopy representations}

Let $G$ be a finite group and $\mathcal{F}$ be a family of subgroups of $G$ that is closed under conjugations and taking subgroups. The orbit category $\operatorname{Or}_{\mathcal{F}} G$ is defined as the category whose objects are orbits of type $G / K$, with $K \in \mathcal{F}$, and where the morphisms from $G / K$ to $G / L$ are given by $G$-maps:

$$
\operatorname{Mor}_{\mathrm{Or}_{\mathcal{F}} G}(G / K, G / L)=\operatorname{Map}_{G}(G / K, G / L) .
$$

The category $\Gamma_{G}=\mathrm{Or}_{\mathcal{F}} G$ is a small category, and we can consider the module category over $\Gamma_{G}$ in the following sense. Let $R$ be a commutative ring with 1. A (right) $R \Gamma_{G}$-module $M$ is a contravariant functor from $\Gamma_{G}$ to the category of $R$-modules. We 
denote the $R$-module $M(G / K)$ simply by $M(K)$ and write $M(f): M(L) \rightarrow M(K)$ for a $G$-map $f: G / K \rightarrow G / L$.

The category of $R \Gamma_{G}$-modules is an abelian category, so the usual concepts of homological algebra, such as kernel, direct sum, exactness, projective module, etc., exist for $R \Gamma_{G}$-modules. Note that an exact sequence of $R \Gamma_{G}$-modules $0 \rightarrow A \rightarrow B \rightarrow$ $C \rightarrow 0$ is exact if and only if

$$
0 \rightarrow A(K) \rightarrow B(K) \rightarrow C(K) \rightarrow 0
$$

is an exact sequence of $R$-modules for every $K \in \mathcal{F}$. For an $R \Gamma_{G}$-module $M$ the $R$ module $M(K)$ can also be considered as an $R W_{G}(K)$-module in an obvious way where $W_{G}(K)=N_{G}(K) / K$. We will follow the convention in [10] and consider $M(K)$ as a right $R W_{G}(K)$-module. In particular, we will consider the sequence above as an exact sequence of right $R W_{G}(K)$-modules.

For each $H \in \mathcal{F}$, let $F_{H}:=R\left[G / H^{?}\right]$ denote the $R \Gamma_{G}$-module with values $F_{H}(K)=$ $R\left[(G / H)^{K}\right]$ for every $K \in \mathcal{F}$, and where for every $G$-map $f: G / L \rightarrow G / K$, the induced map $F_{H}(f): R\left[(G / H)^{K}\right] \rightarrow R\left[(G / H)^{L}\right]$ is defined in the obvious way. By the Yoneda lemma, there is an isomorphism

$$
\operatorname{Hom}_{R \Gamma_{G}}\left(R\left[G / H^{?}\right], M\right) \cong M(H)
$$

for every $R \Gamma_{G}$-module $M$. From this it is easy to show that the module $R\left[G / H^{?}\right]$ is a projective module in the usual sense, for each $H \in \mathcal{F}$. An $R \Gamma_{G}$-module is called free if it is isomorphic to a direct sum of $R \Gamma_{G}$-modules of the form $R\left[G / H^{\text {? }}\right]$. It can be shown that an $R \Gamma_{G}$-module is projective if and only if it is a direct summand of a free module. Further details about the properties of modules over the orbit category can be found in $[\mathbf{8}]$ (see also Lück $[\mathbf{1 0}, \S 9, \S 17]$ and tom Dieck $[\mathbf{1 2}, \S 10-11]$ ).

In this section we consider chain complexes $\mathbf{C}$ of $R \Gamma_{G}$-modules, with respect to a given family $\mathcal{F}$. When we say a chain complex we always mean a non-negative complex, so $\mathbf{C}_{i}=0$ for $i<0$. We call a chain complex $\mathbf{C}$ projective (resp. free) if for all $i \geqslant 0$, the modules $\mathbf{C}_{i}$ are projective (resp. free). We say that a chain complex $\mathbf{C}$ is finite if $\mathbf{C}_{i}=0$ for all $i>n$, for some $n \geqslant 0$, and the chain modules $\mathbf{C}_{i}$ are all finitely generated $R \Gamma_{G}$-modules.

Remark 2.1. Up to chain homotopy equivalence, there is no difference between finite projective chain complexes and finite-dimensional projective chain complexes with finitely generated homology (see $[\mathbf{9}, 3.6])$. For this reason, our definitions and results are mostly stated for finite chain complexes.

We define the support of a chain complex $\mathbf{C}$ over $R \Gamma_{G}$ as the family of subgroups

$$
\operatorname{Supp}(\mathbf{C})=\{H \in \mathcal{F} \mid \mathbf{C}(H) \neq 0\} .
$$

It is sometimes convenient to vary the family of subgroups.

Definition 2.2. If $\mathcal{F} \subset \mathcal{G}$ are two families, the orbit category $\Gamma_{G, \mathcal{F}}=\mathrm{Or}_{\mathcal{F}} G$ is a full-subcategory of $\Gamma_{G, \mathcal{G}}=\operatorname{Or}_{\mathcal{G}} G$. If $M$ is a module over $R \Gamma_{G, \mathcal{F}}$, then we define $\operatorname{Inc}_{\mathcal{F}}^{\mathcal{G}}(M)(H)=M(H)$ if $H \in \mathcal{F}$, and zero otherwise. Similarly, for a module $N$ over $R \Gamma_{G, \mathcal{G}}$, define $\operatorname{Res}_{\mathcal{F}}^{\mathcal{S}}(N)(H)=N(H)$, for $H \in \mathcal{F}$. We extend to maps and chain complexes in a similar way. Note that $\operatorname{Supp}\left(\operatorname{Inc}_{\mathcal{F}}^{\mathcal{G}}(\mathbf{C})\right)=\operatorname{Supp}(\mathbf{C})$, and $\operatorname{Supp}\left(\operatorname{Res}_{\mathcal{F}}^{\mathcal{G}}(\mathbf{D})\right)=$ $\operatorname{Supp}(\mathbf{D}) \cap \mathcal{F}$. 
Given a $G$-CW-complex $X$, there is an associated chain complex of $R \Gamma_{G}$-modules over the family of all subgroups

$$
\mathbf{C}\left(X^{?} ; R\right): \quad \cdots \rightarrow R\left[X_{n}{ }^{?}\right] \stackrel{\partial_{n}}{\longrightarrow} R\left[X_{n-1}{ }^{?}\right] \rightarrow \cdots \stackrel{\partial_{1}}{\longrightarrow} R\left[X_{0}{ }^{?}\right] \rightarrow 0,
$$

where $X_{i}$ denotes the set of (oriented) $i$-dimensional cells in $X$ and $R\left[X_{i}{ }^{?}\right]$ is the $R \Gamma_{G^{-}}$ module defined by $R\left[X_{i}{ }^{?}\right](H)=R\left[X_{i}^{H}\right]$ for every $H \leqslant G$. We denote the homology of this complex by $H_{*}\left(X^{?} ; R\right)$. The chain complex $\mathbf{C}\left(X^{H} ; R\right)$ is actually defined for all subgroups $H \leqslant G$, but for a given family of subgroups $\mathcal{F}$, we can restrict its values from $\operatorname{Or}(G)$ to the full subcategory $\operatorname{Or}_{\mathcal{F}} G$.

The smallest family containing all the isotropy subgroups $\left\{G_{x} \mid x \in X\right\}$ is

$$
\mathfrak{I s o}(X)=\left\{H \leqslant G \mid X^{H} \neq \emptyset\right\},
$$

and this motivates our notion of support for algebraic chain complexes. In particular, we have

$$
\operatorname{Supp}\left(\operatorname{Res} \mathcal{F}\left(\mathbf{C}\left(X^{?} ; R\right)\right)\right)=\mathcal{F} \cap \mathfrak{I s o}(X) .
$$

If the family $\mathcal{F}$ includes all the isotropy subgroups of $X$, then the complex $\mathbf{C}\left(X^{?} ; R\right)$ is a chain complex of free $R \Gamma_{G}$-modules, hence projective $R \Gamma_{G}$-modules, but otherwise the chain modules may not be projective over $R \Gamma_{G}$.

Given a finite-dimensional $G$-CW-complex $X$, there is a dimension function

$$
\operatorname{Dim} X: \mathcal{S}(G) \rightarrow \mathbb{Z}
$$

given by $(\operatorname{Dim} X)(H)=\operatorname{dim} X^{H}$ for all $H \in \mathcal{S}(G)$, where $\mathcal{S}(G)$ denote the set of all subgroups of $G$. By convention, we set $\operatorname{dim} \emptyset=-1$ for the dimension of the empty set. In a similar way, we define the following:

Definition 2.3. The (chain) dimension function of a finite-dimensional chain complex $\mathbf{C}$ over $R \Gamma_{G}$ is defined as the function $\operatorname{Dim} \mathbf{C}: \mathcal{S}(G) \rightarrow \mathbb{Z}$ which has the value

$$
(\operatorname{Dim} \mathbf{C})(H)=\operatorname{dim} \mathbf{C}(H)
$$

for all $H \in \mathcal{F}$, where the dimension of a chain complex of $R$-modules is defined as the largest integer $d$ such $C_{d} \neq 0$ (hence the zero complex has dimension -1 ). If $H \notin \mathcal{F}$, then we set $(\operatorname{Dim} \mathbf{C})(H)=-1$.

The dimension function $\operatorname{Dim} \mathbf{C}: \mathcal{S}(G) \rightarrow \mathbb{Z}$ is conjugation invariant, meaning that it takes the same value on conjugate subgroups of $G$. The term super class function is often used for such functions.

Definition 2.4. The support of a super class function $\underline{n}$ is defined as the set

$$
\operatorname{Supp}(\underline{n})=\{H \leqslant G: \underline{n}(H) \neq-1\} .
$$

We say that a super class function $\underline{n}: \mathcal{S}(G) \rightarrow \mathbb{Z}$ is supported on $\mathcal{F}$ if $\operatorname{Supp}(\underline{n}) \subseteq \mathcal{F}$. Note that $\operatorname{Supp}(\mathbf{C}) \subseteq \mathcal{F}$ is the support of the dimension function $\operatorname{Dim} \mathbf{C}$ of a chain complex $\mathbf{C}$ over $R \Gamma_{G}$.

In a similar way, we can define the homological dimension function of a chain complex $\mathbf{C}$ of $R \Gamma_{G}$-modules as the function HomDim $\mathbf{C}: \mathcal{S}(G) \rightarrow \mathbb{Z}$, where for each 
$H \in \mathcal{F}$, the integer

$$
(\operatorname{HomDim} \mathbf{C})(H)=\operatorname{hdim} \mathbf{C}(H)
$$

is defined as the largest integer $d$ such that $H_{d}(\mathbf{C}(H)) \neq 0$. If $H \notin \mathcal{F}$, then we set $\underline{n}(H)=-1$, as before.

Let us write $(H) \leqslant(K)$ whenever $g^{-1} H g \leqslant K$ for some $g \in G$. Here $(H)$ denotes the set of subgroups conjugate to $H$ in $G$. The notation $(H)<(K)$ means that $(H) \leqslant(K)$ but $(H) \neq(K)$.

Definition 2.5. We call a function $\underline{n}: \mathcal{S}(G) \rightarrow \mathbb{Z}$ monotone if it satisfies the property that $\underline{n}(H) \geqslant \underline{n}(K)$ whenever $(H) \leqslant(K)$. We say that a monotone function $\underline{n}$ is strictly monotone if $\underline{n}(H)>\underline{n}(K)$, whenever $(H)<(K)$.

We have the following:

Lemma 2.6. The (chain) dimension function of every finite-dimensional projective chain complex $\mathbf{C}$ of $R \Gamma_{G}$-modules is monotone.

Proof. Let $(L) \leqslant(K)$. If $\underline{n}(K)=-1$, then the inequality $\underline{n}(L) \geqslant \underline{n}(K)$ is clear. So assume $\underline{n}(K)=n \neq-1$. Then $\mathbf{C}_{n}(K) \neq 0$. By the decomposition theorem for projective $R \Gamma_{G}$-modules [12, Chap. I, Theorem 11.18], every projective $R \Gamma_{G}$-module $P$ is of the form $P \cong \oplus_{H} E_{H} P_{H}$, where $H \in \mathcal{F}$ and $P_{H}$ is a projective $N_{G}(H) / H$-module. Here the $R \Gamma_{G}$-module $E_{H} P_{H}$ is defined by

$$
E_{H} P_{H}(?)=P_{H} \otimes_{R N_{G}(H) / H} \operatorname{RMap}_{G}(G / ?, G / H) .
$$

Applying this decomposition theorem to $\mathbf{C}_{n}$, we observe that $\mathbf{C}_{n}$ must have a summand $E_{H} P_{H}$ with $(K) \leqslant(H)$. But then $\mathbf{C}_{n}(L) \neq 0$, and hence $\underline{n}(L) \geqslant \underline{n}(K)$.

We are particularly interested in chain complexes that have the homology of a sphere when evaluated at every $K \in \mathcal{F}$. To specify the restriction maps in dimension zero, we will consider chain complexes $\mathbf{C}$ that are equipped with an augmentation map $\varepsilon: \mathbf{C}_{0} \rightarrow \underline{R}$ such that $\varepsilon \circ \partial_{1}=0$. Here $\underline{R}$ denotes the constant functor, and we assume that $\varepsilon(H)$ is surjective for $H \in \operatorname{Supp}(\mathbf{C})$. We often consider $\varepsilon$ as a chain map $\mathrm{C} \rightarrow \underline{R}$ by considering $\underline{R}$ as a chain complex over $R \Gamma_{G}$ that is concentrated at zero. We denote a chain complex with an augmentation as a pair $(\mathbf{C}, \varepsilon)$.

By the reduced homology of a complex $(\mathbf{C}, \varepsilon)$, we always mean the homology of the augmented chain complex

$$
\widetilde{\mathbf{C}}=\left\{\cdots \rightarrow \mathbf{C}_{n} \stackrel{\partial_{n}}{\longrightarrow} \cdots \rightarrow \mathbf{C}_{2} \stackrel{\partial_{2}}{\longrightarrow} \mathbf{C}_{1} \stackrel{\partial_{1}}{\longrightarrow} \mathbf{C}_{0} \stackrel{\varepsilon}{\rightarrow} \underline{R} \rightarrow 0\right\},
$$

where $\underline{R}$ is considered to be at dimension -1 . Note that the complex $\widetilde{\mathbf{C}}$ is the -1 shift of the mapping cone of the chain map $\varepsilon: \mathbf{C} \rightarrow \underline{R}$.

Definition 2.7. Let $\underline{n}$ be a super class function supported on $\mathcal{F}$, and let $\mathbf{C}$ be a chain complex over $R \Gamma_{G}$ with respect to a family $\mathcal{F}$ of subgroups.

(i) We say that $\mathbf{C}$ is an $R$-homology $\underline{n}$-sphere if there is an augmentation map $\varepsilon: \mathbf{C} \rightarrow \underline{R}$ such that the reduced homology of $\mathbf{C}(K)$ is the same as the homology of an $\underline{n}(\bar{K})$-sphere (with coefficients in $R$ ) for all $K \in \mathcal{F}$.

(ii) We say that $\mathbf{C}$ is oriented if the $W_{G}(K)$-action on the homology of $\mathbf{C}(K)$ is trivial for all $K \in \mathcal{F}$. 
Note that we do not assume that the dimension function is strictly monotone as in Definition II.10.1 in [12].

In transformation group theory, a $G$-CW-complex $X$ is called a homotopy representation if $X^{H}$ is homotopy equivalent to the sphere $S^{\underline{n}(H)}$, where $\underline{n}(H)=\operatorname{dim} X^{H}$ for every $H \leqslant G$ (see tom Dieck [12, Section II.10]). We now introduce an algebraic analogue of this useful notion for chain complexes over the orbit category.

In [12, II.10], there is a list of properties that are satisfied by homotopy representations. We will use algebraic versions of these properties to define an analogous notion for chain complexes.

Definition 2.8. Let $\mathbf{C}$ be a finite projective chain complex over $R \Gamma_{G}$, which is an $R$-homology $\underline{n}$-sphere. We say $\mathbf{C}$ is an algebraic homotopy representation (over $R$ ) if

(i) The function $\underline{n}$ is a monotone function.

(ii) If $H, K \in \mathcal{F}$ are such that $n=\underline{n}(K)=\underline{n}(H)$, then for every $G$-map $f: G / H \rightarrow$ $G / K$ the induced map $\mathbf{C}(f): \mathbf{C}(K) \rightarrow \mathbf{C}(H)$ is an $R$-homology isomorphism.

(iii) Suppose $H, K, L \in \mathcal{F}$ are such that $H \leqslant K, L$, and let $M=\langle K, L\rangle$ be the subgroup of $G$ generated by $K$ and $L$. If $n=\underline{n}(H)=\underline{n}(K)=\underline{n}(L)>-1$, then $M \in \mathcal{F}$ and $n=\underline{n}(M)$.

Note that conditions (ii) and (iii) of Definition 2.8 are automatic if the dimension function $\underline{n}$ is strictly monotone. Under condition (iii), the isotropy family $\mathcal{F}$ has an important maximality property.

Proposition 2.9. Let $\underline{n}$ be a super class function, and let $\mathbf{C}$ be a projective chain complex of $R \Gamma_{G}$-modules, which is an $R$-homology $\underline{n}$-sphere. If condition (iii) holds, then for each $H \in \mathcal{F}$, the set of subgroups

$$
\mathcal{F}_{H}=\{K \in \mathcal{F} \mid(H) \leqslant(K), \underline{n}(K)=\underline{n}(H)>-1\}
$$

has a unique maximal element, up to conjugation.

Proof. Clear by induction from the statement of condition (iii).

In the remainder of this section we will assume that $R$ is a principal ideal domain. The important examples for us are $R=\mathbb{Z}_{(p)}$ or $R=\mathbb{Z}$. The main result of this section is the following proposition.

Proposition 2.10. Let $\underline{n}$ be a super class function, and let $\mathbf{C}$ be a finite projective chain complex over $R \Gamma_{G}$, which is an $R$-homology $\underline{n}$-sphere. Assume that $R$ is a principal ideal domain. If the equality $\underline{n}=\operatorname{Dim} \mathbf{C}$ holds, then $\mathbf{C}$ is an algebraic homotopy representation.

Before we prove Proposition 2.10, we make some observations and give some definitions for projective chain complexes.

Lemma 2.11. Let $\mathbf{C}$ be a projective chain complex over $R \Gamma_{G}$. Then, for every $G$-map $f: G / H \rightarrow G / K$, the induced map $\mathbf{C}(f): \mathbf{C}(K) \rightarrow \mathbf{C}(H)$ is an injective map with an $R$-torsion-free cokernel. 
Proof. It is enough to show that if $P$ a projective $R \Gamma_{G}$-module, then for every $G$-map $f: G / H \rightarrow G / K$, the induced map $P(f): P(K) \rightarrow P(H)$ is an injective map with a torsion-free cokernel. Since every projective module is a direct summand of a free module, it is enough to prove this for a free module $P=R\left[X^{\text {? }}\right]$, where $X$ is a finite $G$-set. Let $f: G / H \rightarrow G / K$ be the $G$-map defined by $f(H)=g K$. Then the induced map $P(f): R\left[X^{K}\right] \rightarrow R\left[X^{H}\right]$ is the linearization of the map $X^{K} \rightarrow X^{H}$ given by $x \mapsto g x$. Since this map is one-to-one, we can conclude that $P(f)$ is injective with torsion-free cokernel.

When $H \leqslant K$ and $f: G / H \rightarrow G / K$ is the $G$-map defined by $f(g H)=g K$ for each $g \in G$, then we denote the induced map $\mathbf{C}(f): \mathbf{C}(K) \rightarrow \mathbf{C}(H)$ by $r_{H}^{K}$ and call it the restriction map. When $H$ and $K$ are conjugate, so that $K=x^{-1} H x$ for some $x \in G$, then the map $\mathbf{C}(f): \mathbf{C}(K) \rightarrow \mathbf{C}(H)$ induced by the $G$-map $f: G / H \rightarrow G / K$ defined by $f(g H)=g x K$ for each $g \in G$ is called the conjugation map and usually denoted by $c_{K}^{g}$. Note that every $G$-map can be written as a composition of two $G$-maps of the above two types, so every induced map $\mathbf{C}(f): \mathbf{C}(K) \rightarrow \mathbf{C}(H)$ can be written as a composition of restriction and conjugation maps.

Since conjugation maps have inverses, they are always isomorphisms. Therefore, the condition (ii) of Definition 2.8 is actually a statement only about restriction maps. To study the restriction maps more closely, we consider the image of $r_{H}^{K}: \mathbf{C}(K) \rightarrow$ $\mathbf{C}(H)$ for a pair $H \leqslant K$ and denote it by $\mathbf{C}_{H}^{K}$. Note that $\mathbf{C}_{H}^{K}$ is a subcomplex of $\mathbf{C}(H)$ as a chain complex of $R$-modules. Also note that if $\mathbf{C}$ is a projective chain complex, then $\mathbf{C}_{H}^{K}$ is isomorphic to $\mathbf{C}(K)$, as a chain complex of $R$-modules, by Lemma 2.11 .

Lemma 2.12. Let $\mathbf{C}$ be a projective chain complex over $R \Gamma_{G}$. Suppose that $K, L \in \mathcal{F}$ are such that $H \leqslant K$ and $H \leqslant L$, and let $M=\langle K, L\rangle$ be the subgroup generated by $K$ and L. If $\mathbf{C}_{H}^{K} \cap \mathbf{C}_{H}^{L} \neq 0$ then $M \in \mathcal{F}$, and hence we have $\mathbf{C}_{H}^{K} \cap \mathbf{C}_{H}^{L}=\mathbf{C}_{H}^{M}$.

Proof. As before, it is enough to prove this for a free $R \Gamma_{G}$-module $P=R\left[X^{\text {? }}\right]$, where $X$ is a finite $G$-set whose isotropy subgroups lie in $\mathcal{F}$. Note that the restriction maps $r_{H}^{K}$ and $r_{H}^{L}$ are linearizations of the maps $X^{K} \rightarrow X^{H}$ and $X^{L} \rightarrow X^{H}$, respectively, which are defined by inclusion of subsets. Then it is clear that the intersection of images of $r_{H}^{K}$ and $r_{H}^{L}$ would be $R\left[X^{K} \cap X^{L}\right]$ considered as an $R$-submodule of $R\left[X^{H}\right]$. We have $X^{K} \cap X^{L}=X^{M}$, where $M=\langle K, L\rangle$. Therefore, if $\mathbf{C}_{H}^{K} \cap \mathbf{C}_{H}^{L} \neq 0$, then we must have $X^{M} \neq \emptyset$ which implies that $M \in \mathcal{F}$. Thus, $\mathbf{C}_{H}^{M}$ is defined and we can write $\mathbf{C}_{H}^{K} \cap \mathbf{C}_{H}^{L}=\mathbf{C}_{H}^{M}$ by the above fixed point formula.

Now, we are ready to prove Proposition 2.10.

Proof of Proposition 2.10. The first condition in Definition 2.8 follows from Lemma 2.6. For (ii) and (iii), we use the arguments similar to the arguments given in II.10.12 and II.10.13 in [12].

To prove (ii), let $f: G / H \rightarrow G / K$ be a $G$-map. By Lemma 2.11, the induced map $\mathbf{C}(f): \mathbf{C}(K) \rightarrow \mathbf{C}(H)$ is injective with torsion-free cokernel. Let $\mathbf{D}$ denote the cokernel of $\mathbf{C}(f)$. Then we have a short exact sequence of $R$-modules

$$
0 \rightarrow \mathbf{C}(K) \rightarrow \mathbf{C}(H) \rightarrow \mathbf{D} \rightarrow 0,
$$

where both $\mathbf{C}(K)$ and $\mathbf{C}(H)$ have dimension $n$. Now consider the long exact reduced 
homology sequence (with coefficients in $R$ ) associated to this short exact sequence:

$$
\cdots \rightarrow 0 \rightarrow H_{n+1}(\mathbf{D}) \rightarrow H_{n}(\mathbf{C}(K)) \stackrel{f^{*}}{\rightarrow} H_{n}(\mathbf{C}(H)) \rightarrow H_{n}(\mathbf{D}) \rightarrow \cdots .
$$

Note that $\mathbf{D}$ has dimension less than or equal to $n$, so $H_{n+1}(\mathbf{D})=0$ and $H_{n}(\mathbf{D})$ is torsion-free. Since $H_{n}(\mathbf{C}(K))=H_{n}(\mathbf{C}(H))=R$, we obtain that $f^{*}$ is an isomorphism. Since both $\mathbf{C}(K)$ and $\mathbf{C}(H)$ have no other reduced homology, we conclude that $\mathbf{C}(f)$ induces an $R$-homology isomorphism between associated augmented complexes. Since the induced map $\underline{R}(f): \underline{R}(K) \rightarrow \underline{R}(H)$ is the identity map id: $R \rightarrow R$, the chain map $\mathbf{C}(f): \mathbf{C}(K) \rightarrow \mathbf{C}(H)$ is an $R$-homology isomorphism.

To prove (iii), observe that there is a Mayer-Vietoris-type exact sequence associated to the pair of complexes $\mathbf{C}_{H}^{K}$ and $\mathbf{C}_{H}^{L}$ that gives an exact sequence of the form

$$
0 \rightarrow H_{n}\left(\mathbf{C}_{H}^{K} \cap \mathbf{C}_{H}^{L}\right) \rightarrow H_{n}\left(\mathbf{C}_{H}^{K}\right) \oplus H_{n}\left(\mathbf{C}_{H}^{L}\right) \rightarrow H_{n}\left(\mathbf{C}_{H}^{K}+\mathbf{C}_{H}^{L}\right) \rightarrow H_{n-1}\left(\mathbf{C}_{H}^{K} \cap \mathbf{C}_{H}^{L}\right) \rightarrow 0 .
$$

Here we again take the homology sequence as the reduced homology sequence.

Let $i^{K}: \mathbf{C}_{H}^{K} \rightarrow \mathbf{C}(H), i_{H}^{L}: \mathbf{C}_{H}^{L} \rightarrow \mathbf{C}(H)$, and $j: \mathbf{C}_{H}^{K}+\mathbf{C}_{H}^{L} \rightarrow \mathbf{C}(H)$ denote the inclusion maps. We have zero on the left-most term since $\mathbf{C}_{H}^{K}+\mathbf{C}_{H}^{L}$ is an $n$-dimensional complex. To see the zero on the right-most term, note that by Lemma $2.11, \mathbf{C}_{H}^{K} \cong$ $\mathbf{C}(K)$ and $\mathbf{C}_{H}^{L} \cong \mathbf{C}(L)$ as chain complexes of $R$-modules, so they have the same homology. This gives that $H_{i}\left(\mathbf{C}_{H}^{K}\right)=H_{i}\left(\mathbf{C}_{H}^{L}\right)=0$ for $i \leqslant n-1$.

Also note that by part (ii), the composition

$$
H_{n}(\mathbf{C}(K)) \cong H_{n}\left(\mathbf{C}_{H}^{K}\right) \stackrel{i_{*}^{K}}{\longrightarrow} H_{n}\left(\mathbf{C}_{H}^{K}+\mathbf{C}_{H}^{L}\right) \stackrel{j_{*}}{\longrightarrow} H_{n}(\mathbf{C}(H))
$$

is an isomorphism. So $j_{*}$ is surjective. Since $H_{n+1}\left(\mathbf{C}(H) /\left(\mathbf{C}_{H}^{K}+\mathbf{C}_{H}^{L}\right)\right)=0$, we see that $j_{*}$ is also injective. Therefore, $j_{*}$ is an isomorphism. This implies that $i_{*}^{K}$ is an isomorphism. Similarly one can show that $i_{*}^{L}: H_{n}\left(\mathbf{C}_{H}^{L}\right) \rightarrow H_{n}\left(\mathbf{C}_{H}^{K}+\mathbf{C}_{H}^{L}\right)$ is also an isomorphism. Using these isomorphisms and looking at the exact sequence above, we conclude that $H_{n}\left(\mathbf{C}_{H}^{K} \cap \mathbf{C}_{H}^{L}\right) \cong R$ and $H_{i}\left(\mathbf{C}_{H}^{K} \cap \mathbf{C}_{H}^{L}\right)=0$ for $i \leqslant n-1$. Therefore, $\mathbf{C}_{H}^{K} \cap \mathbf{C}_{H}^{L}$ is an $R$-homology $n$-sphere.

Since $n>-1$, this implies that $\mathbf{C}_{H}^{K} \cap \mathbf{C}_{H}^{L} \neq 0$, and hence $M=\langle K, L\rangle \in \mathcal{F}$ by Lemma 2.12. Moreover, $\mathbf{C}_{H}^{K} \cap \mathbf{C}_{H}^{L}=\mathbf{C}_{H}^{M}$. This proves that $\underline{n}(M)=n$, as desired.

\section{The Proof of Theorem A}

In this section we will again assume that $R$ is a principal ideal domain. The main examples for us are $R=\mathbb{Z}_{(p)}$ or $R=\mathbb{Z}$, as before.

Definition 3.1. We say a chain complex $\mathbf{C}$ of $R \Gamma_{G}$-modules is tight at $H \in \mathcal{F}$ if

$$
\operatorname{dim} \mathbf{C}(H)=\operatorname{hdim} \mathbf{C}(H) .
$$

We call a chain complex of $R \Gamma_{G}$-modules tight if it is tight at every $H \in \mathcal{F}$.

Suppose that $\mathbf{C}$ is a finite projective complex over $R \Gamma_{G}$, which is an $R$-homology $\underline{n}$-sphere. If $\mathbf{C}$ is chain homotopy equivalent to a tight complex, then Proposition 2.10 shows that $\mathbf{C}$ is an algebraic homotopy representation. This establishes one direction of Theorem A. The other direction uses the assumption that the chain modules of $\mathbf{C}$ are free over $R \Gamma_{G}$. 
Theorem 3.2. Let $\mathbf{C}$ be a finite chain complex of free $R \Gamma_{G}$-modules, which is a homology $\underline{n}$-sphere. If $\mathbf{C}$ is an algebraic homotopy representation over $R$, then $\mathbf{C}$ is chain homotopy equivalent to a finite free chain complex $\mathbf{D}$ which is tight.

Remark 3.3. If $\mathbf{C}$ is a finite projective chain complex, then the analogous result holds for a sufficiently large $k$-fold join tensor product $\mathbf{C}^{\prime}=*_{k} \mathbf{C}$, by [8, Theorem 7.6].

We need to show that the complex $\mathbf{C}$ can be made tight at each $H \in \mathcal{F}$ by replacing it with a chain complex homotopic to $\mathbf{C}$. The proof is given in several steps.

\subsection{Tightness at maximal isotropy subgroups}

Let $H$ be a maximal element in $\mathcal{F}$. Consider the subcomplex $\mathbf{C}^{(H)}$ of $\mathbf{C}$ formed by free summands of $\mathbf{C}$ isomorphic to $R\left[G / H^{\text {? }}\right]$. Note that $\mathbf{C}^{(H)}$ is a submodule because $\operatorname{Hom}_{R \Gamma_{G}}\left(R\left[G / H^{?}\right], R\left[G / K^{?}\right]\right) \neq 0$ only if $(H) \leqslant(K)$, and since $H$ is maximal, we have $\partial_{i}\left(\mathbf{C}_{i}^{(H)}\right) \subseteq \mathbf{C}_{i-1}^{(H)}$ for all $i$. The complex $\mathbf{C}^{(H)}$ is a complex of isotypic modules of type $R\left[G / H^{?}\right]$. Recall that free $R \Gamma_{G}$-module $F$ is called isotypic of type $G / H$ if it is isomorphic to a direct sum of copies of a free module $R\left[G / H^{?}\right]$, for some $H \in \mathcal{F}$. For extensions involving isotypic modules, we have the following:

Lemma 3.4. Let

$$
\mathcal{E}: 0 \rightarrow F \rightarrow F^{\prime} \rightarrow M \rightarrow 0
$$

be a short exact sequence of $R \Gamma_{G}$-modules such that both $F$ and $F^{\prime}$ are isotypic free modules of the same type $G / H$. If $M(H)$ is R-torsion-free, then $\mathcal{E}$ splits and $M$ is stably free.

Proof. This is Lemma 8.6 of $[\mathbf{8}]$. The assumption that $R$ is a principal ideal domain ensures that finitely generated $R$-torsion-free modules are free.

Note that $\mathbf{C}^{(H)}(H)=\mathbf{C}(H)$, since $H$ is maximal in $\mathcal{F}$. This means that $\mathbf{C}^{(H)}$ is a finite free chain complex over $R \Gamma_{G}$ of the form

$$
\mathbf{C}^{(H)}: 0 \rightarrow F_{d} \rightarrow F_{d-1} \rightarrow \cdots \rightarrow F_{1} \rightarrow F_{0} \rightarrow 0,
$$

which is a $R$-homology $\underline{n}(H)$-sphere, with $\underline{n}(H) \leqslant d$.

Lemma 3.5. Let $\mathbf{C}$ be a finite chain complex of free $R \Gamma_{G}$-modules. Then $\mathbf{C}$ is chain homotopy equivalent to a finite free chain complex $\mathbf{D}$, which is tight at every maximal element $H \in \mathcal{F}$.

Proof. We apply [8, Proposition 8.7] to the subcomplex $\mathbf{C}^{(H)}$, for each maximal element $H \in \mathcal{F}$. The key step is provided by Lemma 3.4.

\subsection{The inductive step}

To make the complex $\mathbf{C}$ tight at every $H \in \mathcal{F}$, we use a downward induction, but the situation at an intermediate step is more complicated than the first step considered above.

Suppose that $H \in \mathcal{F}$ is such that $\mathbf{C}$ is tight at every $K \in \mathcal{F}$ such that $(K)>$ $(H)$. Let $\mathbf{C}^{(H)}$ denote the subcomplex of $\mathbf{C}$ with free summands of type $R\left[G / K^{?}\right]$ satisfying $(H) \leqslant(K)$. In a similar way, we can define the subcomplex $\mathbf{C}^{>(H)}$ of $\mathbf{C}$ 
whose free summands are of type $R\left[G / K^{?}\right]$ with $(H)<(K)$. The complex $\mathbf{C}^{>(H)}$ is a subcomplex of $\mathbf{C}^{(H)}$. Let us denote the quotient complex $\mathbf{C}^{(H)} / \mathbf{C}^{>(H)}$ by $\mathbf{C}_{(H)}$. The complex $\mathbf{C}_{(H)}$ is isotypic with isotropy type $R\left[G / H^{\text {? }}\right]$. We have a short exact sequence of chain complexes of free $R \Gamma_{G}$-modules

$$
0 \rightarrow \mathbf{C}^{>(H)} \rightarrow \mathbf{C}^{(H)} \rightarrow \mathbf{C}_{(H)} \rightarrow 0 .
$$

By evaluating at $H$, we obtain an exact sequence of chain complexes

$$
0 \rightarrow \mathbf{C}^{>(H)}(H) \rightarrow \mathbf{C}^{(H)}(H) \rightarrow \mathbf{C}_{(H)}(H) \rightarrow 0 .
$$

Since $\mathbf{C}^{(H)}(H)=\mathbf{C}(H)$ and the image of the map on the left is generated by summands of the form $R\left[G / K^{?}\right]$ with $(H)<(K)$, the complex $\mathbf{C}_{(H)}(H)$ is isomorphic to $S_{H} \mathbf{C}$ as an $R\left[N_{G}(H) / H\right]$-module. Here $S_{H}$ denotes is splitting functor defined more generally for any module over an EI-category (see [10, Definition 9.26]).

Note that we also have a sequence

$$
0 \rightarrow \mathbf{C}^{(H)} \rightarrow \mathbf{C} \rightarrow \mathbf{C} / \mathbf{C}^{(H)} \rightarrow 0 .
$$

If we can show that $\mathbf{C}^{(H)}$ is homotopy equivalent to a complex $\mathbf{D}^{\prime}$ which is tight at $H$, then by taking the push-out of $\mathbf{D}^{\prime}$ along the injective map $\mathbf{C}^{(H)} \rightarrow \mathbf{C}$, we can find a complex $\mathbf{D}$ homotopy equivalent to $\mathbf{C}$ which is tight at every $K \in \mathcal{F}$ with $(K) \geqslant(H)$. So it is enough to show that $\mathbf{C}^{(H)}$ is homotopy equivalent to a complex $\mathbf{D}^{\prime}$ which is tight at $H$.

Lemma 3.6. Let $\mathbf{C}$ be a finite free chain complex of $R \Gamma_{G}$-modules, such that $\mathbf{C}$ is tight at every $K \in \mathcal{F}$ with $(K)>(H)$, for some $H \in \mathcal{F}$. Suppose

(i) $n=\operatorname{hdim} \mathbf{C}(H) \geqslant \operatorname{dim} \mathbf{C}(K)$, for all $(K)>(H)$, and that

(ii) $H_{n+1}\left(S_{H} \mathbf{C}\right)=0$.

Then $\mathbf{C}^{(H)}$ is homotopy equivalent to a finite free chain complex $\mathbf{D}^{\prime}$, which is tight at every $K \in \mathcal{F}$ with $(K) \geqslant(H)$.

Proof. Let us fix $H \in \mathcal{F}$ and assume that $\mathbf{C}$ is tight at every $K \in \mathcal{F}$ with $(K)>(H)$. We first observe that $\mathbf{C}^{>(H)}$ has dimension $\leqslant n=\operatorname{hdim} \mathbf{C}(H)$, since $\mathbf{C}^{>(H)}(K)=$ $\mathbf{C}(K)$ for $(K)>(H)$, and $\operatorname{dim} \mathbf{C}(K) \leqslant n$. Let $d=\operatorname{dim} \mathbf{C}(H)$. If $d=n$, then we are done, so assume that $d>n$. Then $\operatorname{dim} \mathbf{C}_{(H)}=d$, and $\mathbf{C}_{(H)}$ is a complex of the form

$$
\mathbf{C}_{(H)}: 0 \rightarrow F_{d} \rightarrow F_{d-1} \rightarrow \cdots \rightarrow F_{1} \rightarrow F_{0} \rightarrow 0 .
$$

We claim that the map $\partial_{d}: F_{d} \rightarrow F_{d-1}$ in the above chain complex is injective. Note that since $\mathbf{C}_{(H)}$ is isotypic of type $(H)$, it is enough to show that this map is injective when it is calculated at $H$. To see this, observe that the map $\partial_{d}$ is the same as the map obtained by applying the functor $E_{H}$ to the $N_{G}(H) / H$-homomorphism $\partial_{d}(H): F_{d}(H) \rightarrow F_{d-1}(H)$ (see [10, Lemma 9.31]). Since the functor $E_{H}$ is exact, we have ker $\partial_{d}=E_{H}\left(\operatorname{ker} \partial_{d}(H)\right)$. Hence, if $\partial_{d}(H)$ is injective, then $\partial_{n}$ is injective.

We will show that $H_{d}\left(\mathbf{C}_{(H)}(H)\right)=H_{d}\left(S_{H} \mathbf{C}\right)=0$. To see this, consider the short exact sequence $0 \rightarrow \mathbf{C}^{>(H)}(H) \rightarrow \mathbf{C}(H) \rightarrow S_{H} \mathbf{C} \rightarrow 0$. Since the complex $\mathbf{C}^{>(H)}$ has dimension $\leqslant n$, the corresponding long exact sequence gives that

$$
H_{d}\left(S_{H} \mathbf{C}\right) \cong H_{d}(\mathbf{C}(H))=0
$$

when $d>n+1$. If $d=n+1$, then this is true by assumption (ii) in the lemma. Now 
we apply $\left[\mathbf{8}\right.$, Proposition 8.7] to $\mathbf{C}_{(H)}$ to obtain a tight complex $\mathbf{D}^{\prime \prime} \simeq \mathbf{C}_{(H)}$, and then let $\mathbf{D}^{\prime} \simeq \mathbf{C}^{(H)}$ denote the pull-back of $\mathbf{D}^{\prime \prime}$ along the surjection $\mathbf{C}^{(H)} \rightarrow \mathbf{C}_{(H)}$.

\subsection{Verifying the hypothesis for the inductive step}

To complete the proof of Theorem 3.2, we need to show that the assumptions in Lemma 3.6 hold at an intermediate step of the downward induction. We will make detailed use of the internal homological conditions (i), (ii), and (iii) in Definition 2.8, satisfied by an algebraic homotopy representation $\mathbf{C}$. We proceed as follows:

(1) The dimension assumptions in Lemma 3.6 follow from condition (i), since when $\underline{n}$ is monotone, we have

$$
n:=\operatorname{hdim} \mathbf{C}(H)=\underline{n}(H) \geqslant \underline{n}(K)=\operatorname{hdim} \mathbf{C}(K)=\operatorname{dim} \mathbf{C}(K),
$$

for all $K \in \mathcal{F}$ with $(K)>(H)$.

(2) The assumption that $H_{n+1}\left(S_{H} \mathbf{C}\right)=0$ is established in Corollary 3.9. It follows from the conditions (ii) and (iii) and the Mayer-Vietoris argument given below.

In the rest of the section, we assume that $\mathbf{C}$ is a finite projective chain complex of $R \Gamma_{G}$-modules, which is an $R$-homology $\underline{n}$-sphere, and satisfies the conditions (i), (ii), and (iii) in Definition 2.8. Assume also that $\mathbf{C}$ is tight for all $K \in \mathcal{F}$, with $(K)>(H)$ for some fixed subgroup $H \in \mathcal{F}$. We will say $\mathbf{C}$ is tight above $H$, for short. Let $\mathcal{K}_{H}$ denote the set of all subgroups

$$
\mathcal{K}_{H}=\{K \in \mathcal{F} \mid K>H \text { and } n:=\underline{n}(K)=\underline{n}(H)\} .
$$

Let $\mathbf{C}$ be an algebraic homotopy representation, which is tight above $H$. Let $\mathbf{C}_{H}^{K}$ denote the image of the restriction map

$$
r_{H}^{K}: \mathbf{C}(K) \rightarrow \mathbf{C}(H)
$$

for every $K \in \mathcal{F}$ with $K \geqslant H$. Note that $\mathbf{C}_{H}^{K}$ is a subcomplex of $\mathbf{C}(H)$ and by Lemma 2.11, it is isomorphic to $\mathbf{C}(K)$. By condition (iii) of Definition 2.8, the collection $\mathcal{K}_{H}$ has a unique maximal element $M$. In addition, we have the equality

$$
\mathbf{C}^{>(H)}(H)=\sum_{K \in \mathcal{K}_{H}} \mathbf{C}_{H}^{K}
$$

since $(G / K)^{H}$ is the union of the subspaces $(G / K)^{L}$, with $L>H$ and $(L)=(K)$.

Moreover, if $K \in \mathcal{K}_{H}$, then by condition (ii) the subcomplex $\mathbf{C}_{H}^{K}$ is an $R$-homology $n$-sphere and the map

$$
H_{n}\left(\mathbf{C}_{H}^{M}\right) \rightarrow H_{n}\left(\mathbf{C}_{H}^{K}\right)
$$

induced by the inclusion map $\mathbf{C}_{H}^{M} \hookrightarrow \mathbf{C}_{H}^{K}$ is an isomorphism. More generally, the following also holds.

Lemma 3.7. Let $\mathbf{C}$ be an algebraic homotopy representation which is tight above $H$, for some fixed $H \in \mathcal{F}$, and let $K_{1}, \ldots, K_{m}$ be a set of subgroups in $\mathcal{K}_{H}$. Then the subcomplex $\sum_{i=1}^{m} \mathbf{C}_{H}^{K_{i}}$ is an R-homology $n$-sphere and the map

$$
H_{n}\left(\mathbf{C}_{H}^{M}\right) \rightarrow H_{n}\left(\sum_{i=1}^{m} \mathbf{C}_{H}^{K_{i}}\right)
$$

induced by the inclusion maps is an isomorphism. 
Proof. This follows from the Mayer-Vietoris spectral sequence in algebraic topology (see [4, pp. 166-168]), which computes the homology of a union of spaces $X=\bigcup X_{i}$ in terms of the homology of the subspaces and their intersections. We include a direct argument for the reader's convenience.

The case $m=1$ follows from the remarks above. For $m>1$, we have the following Mayer-Vietoris type long exact sequence

$0 \rightarrow H_{n}\left(\mathbf{D}_{m-1} \cap \mathbf{C}_{H}^{K_{m}}\right) \rightarrow H_{n}\left(\mathbf{D}_{m-1}\right) \oplus H_{n}\left(\mathbf{C}_{H}^{K_{m}}\right) \rightarrow H_{n}\left(\mathbf{D}_{m}\right) \rightarrow H_{n-1}\left(\mathbf{D}_{m-1} \cap \mathbf{C}_{H}^{K_{m}}\right) \rightarrow$, where $\mathbf{D}_{j}=\sum_{i=1}^{j} \mathbf{C}_{H}^{K_{i}}$ for $j=m-1, m$. By the inductive assumption, we know that $\mathbf{D}_{m-1}$ is an $R$-homology $n$-sphere and the map $H_{n}\left(\mathbf{C}_{H}^{M}\right) \rightarrow H_{n}\left(\mathbf{D}_{m-1}\right)$ induced by inclusion is an isomorphism.

Note that

$$
\mathbf{D}_{m-1} \cap \mathbf{C}_{H}^{K_{m}}=\left(\sum_{i=1}^{m-1} \mathbf{C}_{H}^{K_{i}}\right) \cap \mathbf{C}_{H}^{K_{m}}=\sum_{i=1}^{m-1}\left(\mathbf{C}_{H}^{K_{i}} \cap \mathbf{C}_{H}^{K_{m}}\right)=\sum_{i=1}^{m-1} \mathbf{C}_{H}^{\left\langle K_{i}, K_{m}\right\rangle},
$$

where the last equality follows from Lemma 2.12. We can apply Lemma 2.12 here because $\mathbf{C}_{H}^{M} \subseteq \mathbf{C}_{H}^{K}$ for all $K \in \mathcal{K}_{H}$ gives that $\mathbf{C}_{H}^{K_{i}} \cap \mathbf{C}_{H}^{K_{m}} \neq 0$ for $1 \leqslant i \leqslant m-1$. Note that we also obtain $\left\langle K_{i}, K_{m}\right\rangle \in \mathcal{K}_{H}$ for all $i$. Applying our inductive assumption again to these subgroups, we obtain that $\mathbf{D}_{m-1} \cap \mathbf{C}_{H}^{K_{m}}$ is an $R$-homology $n$-sphere and that the map

$$
H_{n}\left(\mathbf{C}_{H}^{M}\right) \rightarrow H_{n}\left(\mathbf{D}_{m-1} \cap \mathbf{C}_{H}^{K_{m}}\right)
$$

induced by inclusion is an isomorphism. This gives that $H_{i}\left(\mathbf{D}_{m}\right)=0$ for $i \leqslant n-1$. We also obtain a commuting diagram

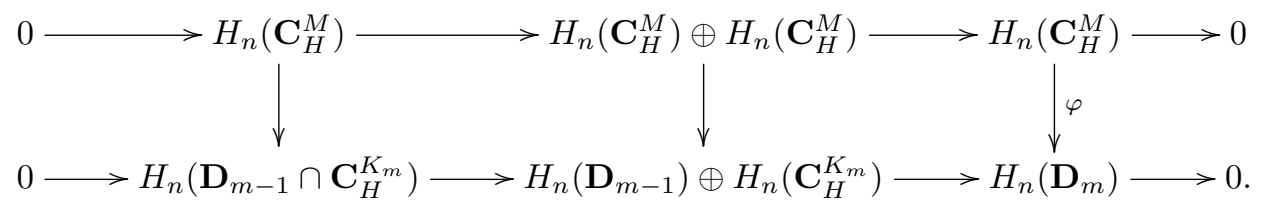

Since all the vertical maps except the map $\varphi$ are known to be isomorphisms, we obtain that $\varphi$ is also an isomorphism by the five lemma. This completes the proof.

Corollary 3.9. Let $\mathbf{C}$ be an algebraic homotopy representation which is tight above $H$, for some fixed $H \in \mathcal{F}$. Then $H_{n+1}\left(S_{H} \mathbf{C}\right)=0$.

Proof. Let $\mathcal{K}_{H}=\left\{K_{1}, \ldots, K_{m}\right\}$. By condition (ii), we know that the composition

$$
H_{n}(\mathbf{C}(M)) \stackrel{\cong}{\rightarrow} H_{n}\left(\mathbf{C}_{H}^{M}\right) \rightarrow H_{n}\left(\sum_{i=1}^{m} \mathbf{C}_{H}^{K_{i}}\right) \rightarrow H_{n}(\mathbf{C}(H))
$$

is an isomorphism. However, we have just proved that the middle map is an isomorphism, and that all the modules involved in the composition are isomorphic to $R$. Therefore, the map induced by inclusion

$$
H_{n}\left(\sum_{i=1}^{m} \mathbf{C}_{H}^{K_{i}}\right) \rightarrow H_{n}(\mathbf{C}(H))
$$

is an isomorphism. Note that if $(H) \leqslant(K)$ and $\underline{n}(K)<n$, for some $K \in \mathcal{F}$, then 
$\operatorname{dim} \mathbf{C}(K)<n$. This means that

$$
H_{n}\left(\mathbf{C}^{>(H)}(H)\right)=H_{n}\left(\sum_{i=1}^{m} \mathbf{C}_{H}^{K_{i}}\right) \cong H_{n}(\mathbf{C}(H)),
$$

where the isomorphism is induced by the the inclusion of chain complexes. From the exact sequence $0 \rightarrow \mathbf{C}^{>(H)}(H) \rightarrow \mathbf{C}(H) \rightarrow S_{H} \mathbf{C} \rightarrow 0$, and the fact that hdim $\mathbf{C}(H)=$ $n$, we conclude that $H_{n+1}\left(S_{H} \mathbf{C}\right)=0$, as required.

This completes the proof of Theorem 3.2 and hence the proof of Theorem A. In [8] we proved the following realization theorem for free $\mathbb{Z} \Gamma_{G}$-module chain complexes, with respect to any family $\mathcal{F}$, which are $\mathbb{Z}$-homology $\underline{n}$-spheres satisfying certain extra conditions.

Theorem 3.10 ([8, Theorem 8.10] and [11]). Let $\mathbf{C}$ be a finite chain complex of free $\mathbb{Z} \Gamma_{G}$-modules, which is a $\mathbb{Z}$-homology $\underline{n}$-sphere. Suppose that $\underline{n}(K) \geqslant 3$ for all $K \in \mathcal{F}$. If $\mathbf{C}_{i}(H)=0$ for all $i>\underline{n}(H)+1$, and all $H \in \mathcal{F}$, then there is a finite $G$ $C W$-complex $X$ with isotropy in $\mathcal{F}$, such that $\mathbf{C}\left(X^{?} ; \mathbb{Z}\right)$ is chain homotopy equivalent to $\mathbf{C}$ as chain complexes of $\mathbb{Z} \Gamma_{G}$-modules.

Note that a homology $\underline{n}$-sphere $\mathbf{C}$ with $\operatorname{Dim} \mathbf{C}=\underline{n}$, and $\underline{n}(K) \geqslant 3$ for all $K \in \mathcal{F}$, will automatically satisfy these conditions. So Corollary B follows immediately from Theorem A and Theorem 3.10.

Remark 3.11. The construction actually produces a finite $G$-CW-complex $X$ with the additional property that all the non-empty fixed sets $X^{H}$ are simply connected. Moreover, by construction, $W_{G}(H)=N_{G}(H) / H$ will act trivially on the homology of $X^{H}$. Therefore, $X$ will be an oriented geometric homotopy representation (in the sense of tom Dieck). From the perspective of Theorem A, since we don't specify any dimension function, a $G$-CW-complex $X$ with all fixed sets $X^{H}$ integral homology spheres will lead (by three-fold join) to a homotopy representation. The same necessary and sufficient conditions for existence apply.

\section{Inflation and deflation of chain complexes}

In this section we define two general operations on chain complexes in preparation for the proof of Theorem C. For a finite $G$-CW complex $X$ that is a mod- $p$ homology sphere, the Borel-Smith conditions can be proved using a reduction argument to certain $p$-group subquotients (compare [12, III.4]). For a subquotient $K / L$, the reduction comes from considering the fixed-point space $X^{L}$ as a $K$-space. To do a similar reduction for chain complexes over $R \Gamma_{G}$, we first introduce a new functor for $R \Gamma_{G}$-modules, called the deflation functor. We will introduce this functor as a restriction functor between corresponding module categories. For this discussion, $R$ can be taken as any commutative ring with 1 , and $\mathcal{F}_{G}$ is any family subject to the extra conditions we assume during the construction.

Let $N$ be a normal subgroup of $G$. We define a functor

$$
F: \Gamma_{G / N} \rightarrow \Gamma_{G}
$$

by considering a $G / N$-set (or $G / N$-map) as a $G$-set (or $G$-map) via composition with the quotient map $G \rightarrow G / N$. For this definition to make sense, the families $\mathcal{F}_{G / N}$ 
and $\mathcal{F}_{G}$ should satisfy the property that if $K \geqslant N$ is such that $(K / N) \in \mathcal{F}_{G / N}$, then $K \in \mathcal{F}_{G}$. Since we always assume the families are nonempty, the above assumption also implies that $N \in \mathcal{F}_{G}$. For notational simplicity from now on, let us denote $K / N$ by $\bar{K}$ for every $K \geqslant N$.

If a family $\mathcal{F}_{G}$ is already given, we will always take $\mathcal{F}_{G / N}=\{\bar{K} \mid K \geqslant N$ and $K \in$ $\left.\mathcal{F}_{G}\right\}$ and the condition above will be automatically satisfied. We also assume that $N \in \mathcal{F}_{G}$ to have a nonempty family for $\mathcal{F}_{G / N}$.

The functor $F$ gives rise to two functors (see [10,9.15]):

$$
\operatorname{Res}_{F}: \operatorname{Mod}-R \Gamma_{G} \rightarrow \operatorname{Mod}-R \Gamma_{G / N}
$$

and

$$
\operatorname{Ind}_{F}: \operatorname{Mod}-R \Gamma_{G / N} \rightarrow \operatorname{Mod}-R \Gamma_{G} .
$$

The first functor, $\operatorname{Res}_{F}$, takes an $R \Gamma_{G}$-module $M$ to the $R \Gamma_{G / N}$-module

$$
\operatorname{Def}_{G / N}^{G}(M):=M \circ F: \Gamma_{G / N} \rightarrow R \text {-Mod. }
$$

We call this functor the deflation functor. Note that

$$
\left(\operatorname{Def}_{G / N}^{G} M\right)(\bar{K})=M(K) .
$$

The induction functor $\operatorname{Inf}_{G / N}^{G}:=\operatorname{Ind}_{F}$ associated to $F$ is called the inflation functor. For every $H \in \mathcal{F}_{G}$, we have

$$
\operatorname{Inf}_{G / N}^{G}(M)(H)=\left(\bigoplus_{\bar{K} \in \mathcal{F}_{G / N}} M(\bar{K}) \otimes_{R W_{\bar{G}}(\bar{K})} R \operatorname{Map}_{G}(G / H, G / K)\right) / \sim,
$$

where the relations come from the tensor product over $R \Gamma_{G / N}$ (see [10, Definition 9.12]). In general, it can be difficult to calculate $\operatorname{Inf}_{G / N}^{G} M$ for an arbitrary $R \Gamma_{G / N^{-}}$ module $M$. In the case where $M$ is a free $R \Gamma_{G / N}$-module, we have the following lemma.

Lemma 4.1. Let $X$ be a finite $G / N$-set. Then we have

$$
\operatorname{Inf}_{G / N}^{G} R\left[X^{?}\right]=R\left[\left(\operatorname{Inf}_{G / N}^{G} X\right)^{?}\right]
$$

Proof. It is enough to show this when $X=\bar{G} / \bar{K}$ for some $K \leqslant G$ such that $K \geqslant N$. In this case, $R\left[(\bar{G} / \bar{K})^{?}\right]$ is isomorphic to $E_{\bar{K}} P_{\bar{K}}$, where $P_{\bar{K}}=R\left[W_{\bar{G}}(\bar{K})\right]$. Since $E_{\bar{K}}(-)$ is defined as induction $\operatorname{Ind}_{F^{\prime}}(-)$ for the functor $F^{\prime}: R\left[W_{\bar{G}}(\bar{K})\right] \rightarrow R \Gamma_{G / N}$ (see $[\mathbf{1 0}$, $9.30]$ ), we have

$$
\operatorname{Inf}_{G / N}^{G} R\left[(\bar{G} / \bar{K})^{?}\right]=\operatorname{Inf}_{G / N}^{G} E_{\bar{K}} P_{\bar{K}}=\operatorname{Ind}_{F} \operatorname{Ind}_{F^{\prime}} P_{\bar{K}}=\operatorname{Ind}_{F \circ F^{\prime}} P_{\bar{K}},
$$

where $F: \Gamma_{G / N} \rightarrow \Gamma_{G}$ is the functor defined above. Since $W_{\bar{G}}(\bar{K}) \cong W_{G}(K)$, after suitable identification, the composition $F \circ F^{\prime}$ becomes the same as the inclusion functor $i: W_{G}(K) \rightarrow \Gamma_{G}$, so we have

$$
\operatorname{Ind}_{F \circ F^{\prime}} P_{\bar{K}}=E_{K} R W_{G}(K)=R\left[G / K^{?}\right],
$$

as desired.

Note that by general properties of restriction and induction functors associated to a functor $F$, the functor $\operatorname{Def}_{G / N}^{G}$ is exact and $\operatorname{Inf}_{G / N}^{G}$ respects projectives (see $[\mathbf{1 0}$, 9.24]). The deflation functor has the following formula for free modules. 
Lemma 4.2. Let $X$ be a $G$-set. Then we have

$$
\operatorname{Def}_{G / N}^{G} R\left[X^{?}\right]=R\left[\left(X^{N}\right)^{?}\right] .
$$

In particular, if $H \in \mathcal{F}_{G}$ implies $H N \in \mathcal{F}_{G}$, then the functor $\operatorname{Def}_{G / N}^{G}$ respects projectives.

Proof. For every $K \in \mathcal{F}_{G}$ such that $K \geqslant N$, we have

$$
\left(\operatorname{Def}_{G / N}^{G} R\left[X^{?}\right]\right)(\bar{K})=R\left[X^{?}\right](K)=R\left[X^{K}\right]=R\left[\left(X^{N}\right)^{K / N}\right]=R\left[\left(X^{N}\right)^{?}\right](\bar{K}) .
$$

Note that $(G / H)^{N}=G / H N$ as a $G / N$-set. If $H \in \mathcal{F}_{G}$ implies $H N \in \mathcal{F}_{G}$, then by assumption $\overline{H N} \in \mathcal{F}_{G / N}$. Hence $R\left[\left((G / H)^{N}\right)^{?}\right]$ is free as an $R \Gamma_{G / N}$-module and $\operatorname{Def}_{G / N}^{G}$ respects projectives.

\section{The Borel-Smith conditions for chain complexes}

Let $G$ be a finite group, and let $X$ be a finite $G$-CW-complex which is a mod- $p$ homology sphere for some prime $p$. Then by Smith theory the fixed point space $X^{H}$ is also a mod- $p$ homology sphere (or empty), for every $p$-subgroup $H \leqslant G$. So if we take $R=\mathbb{Z} / p$ and $\Gamma_{G}$ as the orbit category over the family $\mathcal{F}_{p}$ of all $p$-subgroups of $G$, then the chain complex $\mathbf{C}\left(X^{?} ; \mathbb{Z}\right)$ over $R \Gamma_{G}$ is a finite free chain complex which is an $R$-homology $\underline{n}$-sphere. Here, as before, we take $\underline{n}(H)=-1$ when $X^{H}=\emptyset$. In this case, it is known that the super class function $\underline{n}$ satisfies certain conditions called the Borel-Smith conditions (see [3, Thm. 2.3 in Chapter XIII] or [12, III.5]). These conditions are given as follows.

Definition 5.1. Let $G$ be a finite group, and let $f: \mathcal{S}(G) \rightarrow \mathbb{Z}$ be super class function, where $\mathcal{S}(G)$ denotes the family of all subgroups of $G$. We say the function $f$ satisfies the Borel-Smith conditions at a prime $p$ if it has the following properties:

(i) If $L \triangleleft K \leqslant G$ are such that $K / L \cong \mathbb{Z} / p$, and $p$ is odd, then $f(L)-f(K)$ is even.

(ii) If $L \triangleleft K \leqslant G$ are such that $K / L \cong \mathbb{Z} / p \times \mathbb{Z} / p$, and if $L_{i} / L$ denote the subgroups of order $p$ in $K / L$, then

$$
f(L)-f(K)=\sum_{i=0}^{p}\left(f\left(L_{i}\right)-f(K)\right) .
$$

(iii) If $p=2$, and $L \triangleleft K \triangleleft N \leqslant G$ are such that $L \triangleleft N, K / L \cong \mathbb{Z} / 2$, and $N / L \cong$ $\mathbb{Z} / 4$, then $f(L)-f(K)$ is even.

(iv) If $p=2$, and $L \triangleleft K \triangleleft N \leqslant G$ are such that $L \triangleleft N, K / L \cong \mathbb{Z} / 2$, and $N / L=Q_{8}$ is the quaternion group of order 8 , then $f(L)-f(K)$ is divisible by 4 .

We will show that these conditions are satisfied by the homological dimension function $\underline{n}$ of a finite projective complex $\mathbf{C}$ over $R \Gamma_{G}$ which is an $R$-homology $\underline{n}$ sphere. Recall that $\underline{n}(H)=-1$ whenever $H \notin \mathcal{F}$, by Definition 2.7 .

Theorem C. Let $G$ be a finite group, $R=\mathbb{Z} / p$, and let $\mathcal{F}$ be a given family of subgroups of $G$. If $\mathbf{C}$ is a finite projective chain complex over $R \Gamma_{G}$, which is an $R$ homology $\underline{n}$-sphere, then the function $\underline{n}$ satisfies the Borel-Smith conditions at the prime $p$. 
The rest of the section is devoted to the proof of Theorem C. As a first step of the proof, we extend the given family $\mathcal{F}$ to the family $\mathcal{S}(G)$ of all subgroups of $G$ by taking $\mathbf{C}(H)=0$ for every $H \notin \mathcal{F}$. Note that over the extended family, $\mathbf{C}$ is still a finite projective chain complex over $R \Gamma_{G}$ and an $R$-homology $\underline{n}$-sphere.

The Borel-Smith conditions are conditions on subquotients $K / L$ where $L \triangleleft K \leqslant$ $G$. To show that a Borel-Smith condition holds for a particular subquotient group $K / L$, we consider the complex $\operatorname{Def}_{K / L}^{K} \operatorname{Res}_{K}^{G} \mathbf{C}$ (see Section 4). This is a finite projective complex over $R \Gamma_{K / L}$ because both restriction and deflation functors preserve projectives (note that the condition in Lemma 4.2 is satisfied because we extended our family $\mathcal{F}$ to the family of all subgroups of $G$ ).

Our first observation is the following.

Lemma 5.2. Let $G$ be a finite group, and let $R=\mathbb{Z} / p$. If $\mathbf{C}$ is a finite projective chain complex over $R \Gamma_{G}$, which is an $R$-homology $\underline{n}$-sphere, then whenever $L \triangleleft K \leqslant G$ and $K / L$ is a p-group, we have $\underline{n}(L) \geqslant \underline{n}(K)$.

Proof. By the discussion above, it is enough to show that if $G=\mathbb{Z} / p$ and $\mathbf{C}$ is a finite projective $R \Gamma_{G}$-complex which is an $R$-homology $\underline{n}$-sphere, then the inequality $\underline{n}(1) \geqslant \underline{n}(G)$ holds. Assume that $\underline{n}(1) \neq \underline{n}(G)$. Since $H_{0}=\underline{R}$ is projective, we can add $\mathbf{C}_{-1}=\underline{R}$ and consider the homology of the augmented complex $\widetilde{\mathbf{C}}$. The complex $\widetilde{\mathbf{C}}$ has nontrivial homology only at two dimensions - say, $m$ and $k$ with $m>k$ - so we get an extension of the form

$$
0 \rightarrow H_{m}(\widetilde{\mathbf{C}}) \rightarrow \widetilde{\mathbf{C}}_{m} / \operatorname{im} \partial_{m+1} \rightarrow \cdots \rightarrow \widetilde{\mathbf{C}}_{k+1} \rightarrow \operatorname{ker} \partial_{k} \rightarrow H_{k}(\widetilde{\mathbf{C}}) \rightarrow 0,
$$

where the homology modules are $I_{1} R$ and $I_{G} R$ in some order.

For $H \in \mathcal{F}$, the module $I_{H} M$ denotes the atomic module concentrated at $H$ with the value $\left(I_{H} M\right)(H)=M($ see $[\mathbf{1 0}, 9.29])$. We claim that $H_{m}(\widetilde{\mathbf{C}})=I_{1} R$ and $H_{k}(\widetilde{\mathbf{C}})=$ $I_{G} R$, meaning that the module $I_{G} R$ appears before $I_{1} R$ in the homology. Once we show this, it will imply that $\underline{n}(1)>\underline{n}(G)$, as desired.

Let $\mathbf{D}$ denote the chain complex obtained by erasing the homology groups $H_{m}(\widetilde{\mathbf{C}})$ and $H_{k}(\widetilde{\mathbf{C}})$ from the above exact sequence. Since ker $\partial_{k}$ is projective and im $\partial_{m+1}$ has a finite projective resolution, the ext-group $\operatorname{Ext}_{R \Gamma_{G}}^{*}(\mathbf{D}, M)$ is zero after some fixed dimension, for every $R \Gamma_{G}$-module $M$.

We will take $M=I_{1} R$ for simplicity. Note that the module $I_{1} R$ is concentrated at 1 , so its projective resolution is of the form $E_{1} P_{*}$ for some projective resolution $P_{*}$ of $R$ as an $R G$-module.

There is a two-line spectral sequence $E_{2}^{p, q}=\operatorname{Ext}_{R \Gamma_{G}}^{p}\left(H_{q}(\mathbf{D}), M\right)$ that converges to $\operatorname{Ext}_{R \Gamma_{G}}^{*}(\mathbf{D}, M)$. Suppose, if possible, that $H_{k}(\widetilde{\mathbf{C}})=I_{1} R$. Then the bottom line of this spectral sequence $E_{2}^{*, 0}$ would be isomorphic to

$$
\operatorname{Ext}_{R \Gamma_{G}}^{*}\left(H_{k}(\widetilde{\mathbf{C}}), M\right)=\operatorname{Ext}_{R \Gamma_{G}}^{*}\left(I_{1} R, I_{1} R\right)=H^{i}\left(\operatorname{Hom}_{R \Gamma_{G}}\left(E_{1} P_{*}, I_{1} R\right)\right)=H^{*}(G ; R) .
$$

Since this cohomology ring is not finitely generated, there must be a non-trivial differential from the top line

$$
\operatorname{Ext}_{R \Gamma_{G}}^{*}\left(H_{m}(\widetilde{\mathbf{C}}), M\right)=\operatorname{Ext}_{R \Gamma_{G}}^{*}\left(I_{G} R, I_{1} R\right)
$$

in order for the spectral sequence to converge to a finite-dimensional limit. 
The differential of this spectral sequence is given by multiplication with an extension class in $\operatorname{Ext}_{R \Gamma_{G}}^{m-k+1}\left(I_{1} R, I_{G} R\right)$. But, by a similar calculation as above, we see that

$$
\left.\operatorname{Ext}_{R \Gamma_{G}}^{i}\left(I_{1} R, I_{G} R\right)=H^{i}\left(G,\left(I_{G} R\right)(1)\right)\right)=0
$$

for all $i \geqslant 0$, because $\left(I_{G} R\right)(1)=0$. This contradiction shows that $H_{k}(\widetilde{\mathbf{C}})=I_{G} R$ and $H_{m}(\widetilde{\mathbf{C}})=I_{1} R$, as required.

The above lemma shows that under the conditions of Theorem $\mathrm{C}$, the dimension function $\underline{n}$ is monotone in the sense defined in [12, p. 211]. Now we verify (in separate steps) that the dimension function satisfies the conditions of Definition 5.1. These conditions come from the period of the cohomology of the corresponding subquotient groups.

Lemma 5.3 (Borel-Smith, part (i)). Let $G=\mathbb{Z} / p$, for $p$ an odd prime, let $R=\mathbb{Z} / p$, and let $\mathbf{C}$ be a finite projective $R \Gamma_{G}$-complex which is an $R$-homology $\underline{n-s p h e r e . ~ T h e n ~}$ $\underline{n}(1)-\underline{n}(G)$ is even.

Proof. Consider the subcomplex $\widetilde{\mathbf{C}}^{(G)}$ of $\widetilde{\mathbf{C}}$ consisting of all projectives of type $R\left[G / G^{?}\right]$, and let $\mathbf{D}=\widetilde{\mathbf{C}} / \widetilde{\mathbf{C}}^{(G)}$ denote the quotient complex. The complex $\mathbf{D}$ has nontrivial homology only in dimensions $m$ and $k+1$, where $m=\underline{n}(1)$ and $k=\underline{n}(G)$. Moreover, all the $R \Gamma_{G}$-modules in the complex $\mathbf{D}$ are of the form $R\left[G / 1^{?}\right]$. Evaluating at the subgroup 1, we obtain a chain complex of free $R G$-modules

$$
0 \rightarrow Q_{d} \rightarrow \cdots \rightarrow Q_{m+1} \stackrel{\partial_{m+1}}{\longrightarrow} Q_{m} \rightarrow \cdots \rightarrow Q_{k+1} \stackrel{\partial_{k+1}}{\longrightarrow} Q_{k} \rightarrow \cdots \rightarrow Q_{0} \rightarrow 0
$$

whose homology is $R$ at dimensions $m$ and $k+1$. This gives an exact sequence of the form

$$
0 \rightarrow R \rightarrow Q_{m} / \operatorname{im} \partial_{m+1} \rightarrow \cdots \rightarrow Q_{k+2} \rightarrow \operatorname{ker} \partial_{k+1} \rightarrow R \rightarrow 0 .
$$

Using the fact that free $R G$-modules are both projective and injective, we conclude that all the modules in the above sequence, except the two $R$ 's on both ends, are projective as $R G$-modules, so we have a periodic resolution of length $m-k$. It follows that $m-k=\underline{n}(1)-\underline{n}(G) \equiv 0(\bmod 2)$, since the group $G=\mathbb{Z} / p$ has periodic $R$ cohomology with period 2 .

Remark 5.4. The $R$-cohomology of the group $G=\mathbb{Z} / 2$ is periodic of period 1 .

For condition (ii), the argument is more involved. Note that as before, we may assume that $G=K / L=\mathbb{Z} / p \times \mathbb{Z} / p$ and that $\mathcal{F}=\mathcal{S}(G)$. Since the complex $\mathbf{C}$ is a finite complex of projective modules, for any $R \Gamma_{G}$-module $M$, we have

$$
H^{n}\left(\operatorname{Hom}_{R \Gamma_{G}}(\mathbf{C}, M)\right)=0
$$

for $n>d$, where $d$ is the dimension of the chain complex $\mathbf{C}$. Consider the hypercohomology spectral sequence for the complex $\mathbf{C}$. This is a spectral-sequence with $E_{2}$-term given by

$$
E_{2}^{s, t}=\operatorname{Ext}_{R \Gamma_{G}}^{s}\left(H_{t}(\mathbf{C}), M\right)
$$

which converges to $H^{s+t}\left(\operatorname{Hom}_{R \Gamma_{G}}(\mathbf{C}, M)\right)$. Since $\underline{R}$ is a projective $R \Gamma_{G}$-module (note that $\mathcal{F}_{G}$ is the family of all subgroups of $G$ after the subquotient reduction), we can 
replace $H_{t}(\mathbf{C})$ with the reduced homology $\widetilde{H}_{t}(\mathbf{C})$. Therefore, we have nonzero terms for $E_{2}^{s, t}$ only when $t$ is equal to $n_{1}=\underline{n}(1), n_{G}=\underline{n}(G)$, or $n_{H_{i}}=\underline{n}\left(H_{i}\right)$, where $H_{i}$ are the subgroups of $G$ of order $p$. Since $\underline{n}$ is monotone, we have $n_{1} \geqslant n_{H_{i}} \geqslant n_{G}$ for all $i \in\{0, \ldots, p\}$. The required formula is

$$
n_{1}-n_{G}=\sum_{i=0}^{p}\left(n_{H_{i}}-n_{G}\right) .
$$

Remark 5.6. In the proof below we assume $n_{1}>n_{H_{i}}>n_{G}$ for all $i$, to make the argument easy to follow. If for some $i$ we have $n_{H_{i}}=n_{1}$ or $n_{H_{i}}=n_{G}$, then the argument below can be adjusted easily to include these cases as well.

Note that by adding free summands to the complex $\mathbf{C}$, we can assume that all the cohomology between dimensions $n_{1}$ and $n_{G}$ is concentrated at the dimension $n_{M}=\max _{i}\left\{n_{H_{i}}\right\}$. Then the homology at this dimension will be an $R \Gamma_{G}$-module which is filtered by Heller shifts of homology groups $H_{t}(\mathbf{C})$ at dimensions $t=n_{H_{i}}$ for $i=0, \ldots, p$. Note that homology of the complex $\mathbf{C}$ at dimension $n_{H_{i}}$ is $I_{H_{i}} R$, where $I_{H_{i}} R$ denotes the $R \Gamma_{G}$ module with value $R$ at $H_{i}$ and zero at all the other subgroups. We have the following lemma.

Lemma 5.7. If $i, j \in\{0, \ldots, p\}$ are such that $i \neq j$, then

$$
\operatorname{Ext}_{R \Gamma_{G}}^{m}\left(I_{H_{i}} R, I_{H_{j}} R\right)=0
$$

for every $m \geqslant 0$.

Proof. The projective resolution of $I_{H_{i}}$ is formed by projective modules of type $E_{H} P$ with $H=1$ or $H_{i}$. Since

$$
\operatorname{Hom}_{R \Gamma_{G}}\left(E_{H} P, I_{H_{j}} R\right) \cong \operatorname{Hom}_{R W_{G}(H)}\left(P,\left(I_{H_{j}} R\right)(H)\right)=0
$$

when $i \neq j$, we obtain the desired result.

As a consequence of Lemma 5.7, we conclude that all the extensions in this filtration of $H_{n_{M}}(\mathbf{C})$ are split extensions. Therefore, the homology module $H_{n_{M}}(\mathbf{C})$ is isomorphic to a direct sum of Heller shifts of modules $I_{H_{i}} R$. In particular, we obtain that, for any $R \Gamma_{G}$-module $M$,

$$
\operatorname{Ext}_{R \Gamma_{G}}^{s}\left(H_{n_{M}}(\mathbf{C}), M\right) \cong \oplus_{i} \operatorname{Ext}_{R \Gamma_{G}}^{s+n_{M}-n_{H_{i}}}\left(I_{H_{i}} R, M\right)
$$

for every $s \geqslant 0$.

The spectral sequence given in (5.5) converges to zero for total dimension $>d$. It has only three nonzero horizontal lines, so it gives a long exact sequence of the form

$$
\begin{gathered}
\cdots \rightarrow \operatorname{Ext}_{R \Gamma_{G}}^{k+n_{1}-n_{G}+1}\left(I_{G} R, M\right) \stackrel{\delta}{\rightarrow} \operatorname{Ext}_{R \Gamma_{G}}^{k}\left(I_{1} R, M\right) \stackrel{\gamma}{\rightarrow} \oplus_{i=0}^{p} \operatorname{Ext}_{R \Gamma_{G}}^{k+n_{1}-n_{H_{i}}+1}\left(I_{H_{i}} R, M\right) \\
\rightarrow \operatorname{Ext}_{R \Gamma_{G}}^{k+n_{1}-n_{G}+2}\left(I_{G} R, M\right) \stackrel{\delta}{\rightarrow} \operatorname{Ext}_{R \Gamma_{G}}^{k+1}\left(I_{1} R, M\right) \rightarrow \cdots,
\end{gathered}
$$

where $k$ is an integer such that $k>d-n_{1}$ and $M$ is any $R \Gamma_{G}$-module. If we take $M=I_{1} R$, then $\operatorname{Ext}_{R \Gamma_{G}}^{k}\left(I_{1} R, M\right) \cong H^{k}(G, R)$. When $M=I_{1} R$, the other Ext groups in the above exact sequence also reduce to the cohomology of the group $G$ but with some dimension shifts. 
Lemma 5.8. For every $i \in\{0, \ldots, p\}$, we have

$$
\operatorname{Ext}_{R \Gamma_{G}}^{m}\left(I_{H_{i}} R, I_{1} R\right) \cong \operatorname{Ext}_{R \Gamma_{G}}^{m-1}\left(I_{1} R, I_{1} R\right) \cong H^{m-1}(G ; R)
$$

for every $m \geqslant 1$. We also have

$$
\operatorname{Ext}_{R \Gamma_{G}}^{m}\left(I_{G} R, I_{1} R\right) \cong \oplus_{p} \operatorname{Ext}_{R \Gamma_{G}}^{m-2}\left(I_{1} R, I_{1} R\right) \cong \oplus_{p} H^{m-2}(G ; R)
$$

for every $m \geqslant 2$. Here $\oplus_{p}$ denotes the direct sum of $p$-copies of the same $R$-module.

Proof. Since we already observed that $\operatorname{Ext}_{R \Gamma_{G}}^{k}\left(I_{1} R, I_{1} R\right) \cong H^{k}(G, R)$ for every $k \geqslant 0$, it is enough to show the first isomorphisms. Let $i \in\{0, \ldots, p\}$ and $J_{H_{i}} R$ denote the $R \Gamma_{G}$ module with value $R$ at subgroups 1 and $H_{i}$ and zero at every other subgroup. We assume that the restriction map is the identity. Hence we have a non-split exact sequence of $R \Gamma_{G}$-modules of the form

$$
0 \rightarrow I_{1} R \rightarrow J_{H_{i}} R \rightarrow I_{H_{i}} R \rightarrow 0 .
$$

Note that the projective resolution of $J_{H_{i}} R$ will only include projective modules of the form $E_{H_{i}} P$, so we have $\operatorname{Ext}_{R \Gamma_{G}}^{m}\left(J_{H_{i}} R, I_{1} R\right)=0$ for all $m \geqslant 0$. The long exact Extgroup sequence associated to the above short exact sequence will give the desired isomorphism for the module $I_{H_{i}} R$.

For the second statement in the lemma, we again only need to show that the isomorphism

$$
\operatorname{Ext}_{R \Gamma_{G}}^{m}\left(I_{G} R, I_{1} R\right) \cong \oplus_{p} \operatorname{Ext}_{R \Gamma_{G}}^{m-2}\left(I_{1} R, I_{1} R\right)
$$

holds for all $m \geqslant 2$. Let $N$ denote the $R \Gamma_{G}$-module defined as the kernel of the map $\underline{R} \rightarrow I_{G} R$ which induces the identity homomorphism at $G$. Since the constant module $\underline{R}$ is projective as a $R \Gamma_{G}$-module, we have

$$
\operatorname{Ext}_{R \Gamma}^{m}\left(I_{G} R, I_{1} R\right) \cong \operatorname{Ext}_{R \Gamma}^{m-1}\left(N, I_{1} R\right)
$$

for $m \geqslant 2$. Note that there is an exact sequence of the form

$$
0 \rightarrow \oplus_{p} I_{1} R \rightarrow \oplus_{i=0}^{p} J_{H_{i}} R \rightarrow N \rightarrow 0 .
$$

Since $\operatorname{Ext}_{R \Gamma_{G}}^{m}\left(J_{H_{i}} R, I_{1} R\right)=0$ for all $m \geqslant 0$, we obtain

$$
\operatorname{Ext}_{R \Gamma}^{m}\left(I_{G} R, I_{1} R\right) \cong \operatorname{Ext}_{R \Gamma}^{m-1}\left(N, I_{1} R\right) \cong \oplus_{p} \operatorname{Ext}_{R \Gamma}^{m-2}\left(I_{1} R, I_{1} R\right) \cong \oplus_{p} H^{m-2}(G ; R)
$$

for every $m \geqslant 2$. This completes the proof of the lemma.

Lemma 5.9 (Borel-Smith, part (ii)). Let $G=\mathbb{Z} / p \times \mathbb{Z} / p$, let $R=\mathbb{Z} / p$, and let $\mathbf{C}$ be a finite projective $R \Gamma_{G}$-complex which is an $R$-homology $\underline{n}$-sphere. Then

$$
\underline{n}(1)-\underline{n}(G)=\sum_{i=0}^{p}\left(\underline{n}\left(H_{i}\right)-\underline{n}(G)\right),
$$

where $H_{0}, H_{1}, \ldots, H_{p}$ denote the distinct subgroups of $G$ of order $p$.

Proof. Using the Ext-group calculations given in Lemma 5.8, we obtain a long exact 
sequence of the form

$$
\begin{gathered}
\cdots \rightarrow \oplus_{p} H^{k+n_{1}-n_{G}-1}(G ; R) \stackrel{\delta}{\rightarrow} H^{k}(G ; R) \stackrel{\gamma}{\rightarrow} \oplus_{i=0}^{p} H^{k+n_{1}-n_{H_{i}}}(G ; R) \\
\rightarrow \oplus_{p} H^{k+n_{1}-n_{G}}(G ; R) \stackrel{\delta}{\rightarrow} H^{k+1}(G ; R) \rightarrow \cdots
\end{gathered}
$$

where $k>d-n_{1}$. We claim that the map $\gamma$ is injective. Observe that if $\gamma=\oplus \gamma_{i}$, then for each $i$ the map $\gamma_{i}$ can be defined as multiplication with some cohomology class $u_{i}$. To see this, observe that $\gamma$ is the map induced by the differential

$$
d_{n_{1}-n_{M}+1}: \operatorname{Ext}_{R \Gamma_{G}}^{k}\left(H_{n_{1}}(\mathbf{C}), I_{1} R\right) \rightarrow \operatorname{Ext}_{R \Gamma_{G}}^{k+n_{1}-n_{M}+1}\left(H_{n_{M}}(\mathbf{C}), I_{1} R\right)
$$

on the hypercohomology spectral sequence given at (5.5). This spectral sequence has an $\operatorname{Ext}_{R \Gamma}^{*}\left(I_{1} R, I_{1} R\right)$-module structure where the multiplication is given by the Yoneda product, i.e., by splicing the corresponding extensions (see [2, Section 4]).

Under the isomorphisms given in Lemma 5.8, the differential $d_{n_{1}-n_{M}+1}$ becomes a map $H^{k}(G, R) \rightarrow \oplus_{i} H^{k+n_{1}-n_{H_{i}}}(G, R)$ and the Yoneda product of Ext-groups is the same as the usual cup product multiplication in group cohomology under the canonical isomorphism $\operatorname{Ext}_{R \Gamma_{G}}^{m}\left(I_{1} R, I_{1} R\right) \cong H^{m}(G, R)$ (for comparison of different products on group cohomology, see [5, Proposition 4.3.5]). So we can conclude that $\gamma_{i}$ is the map defined by multiplication (the usual cup product) with a cohomology class $u_{i} \in H^{n_{1}-n_{H_{i}}}(G, R)$.

Suppose now that $\gamma$ is not injective. Note that when $p=2$, the cohomology ring $H^{*}(G, R)$ is isomorphic to a polynomial algebra $R\left[t_{1}, t_{2}\right]$ with $\operatorname{deg} t_{i}=1$ for $i=1,2$. Since there are no nonzero divisors in a polynomial algebra, if $\gamma$ is not injective, then it must be the zero map.

In this case where $p$ is odd, the cohomology ring $H^{*}(G, R)$ is isomorphic to the tensor product of an exterior algebra with a polynomial algebra

$$
\Lambda_{R}\left(a_{1}, a_{2}\right) \otimes R\left[x_{1}, x_{2}\right]
$$

where $\operatorname{deg} a_{i}=1$ and $\operatorname{deg} x_{i}=2$. The nonzero divisors of this ring are multiples of $a_{i}$ or $a_{j}$. For each $i$, the class $u_{i}$ is an even dimensional class, so it must be a multiple of $a_{1} a_{2}$ (note that the class $u_{i}$ has degree $n_{1}-n_{H_{i}}$, which is an even number by Lemma 5.3).

Hence in either case ( $p=2$ or $p$ odd), the restriction of the entire spectral sequence to some $H_{i}$ will result in a spectral sequence with zero differentials. This is because $\operatorname{Res}_{H_{i}}^{G} I_{G} R=0$ and $\operatorname{Res}_{H_{i}}^{G} I_{H_{j}} R=0$ if $i \neq j$. So, if $\gamma$ is not injective, the restriction of the spectral sequence to a subgroup $H_{i}$ gives a spectral sequence that collapses. But the restriction of $\mathbf{C}$ to a proper subgroup is still a finite projective chain complex, so this gives a contradiction. Hence we can conclude that $\gamma$ is injective.

The fact that $\gamma$ is injective gives a short exact sequence of the form

$$
0 \rightarrow H^{k}(G ; R) \stackrel{\gamma}{\rightarrow} \oplus_{i=0}^{p} H^{k+n_{1}-n_{H_{i}}}(G ; R) \rightarrow \oplus_{p} H^{k+n_{1}-n_{G}}(G ; R) \rightarrow 0,
$$

for every $k>d-n_{1}$. Since $\operatorname{dim}_{R} H^{m}(G ; R)=m+1$, we obtain

$$
(k+1)+p\left(k+n_{1}-n_{G}+1\right)=\sum_{i=0}^{p}\left(k+n_{1}-n_{H_{i}}+1\right) .
$$

Cancelling the $(k+1)$ 's and grouping the terms in a different way gives the desired equality. 
The next part uses the same spectral sequence, but the details are much simpler.

Lemma 5.10 (Borel-Smith, part (iii)). Let $G=\mathbb{Z} / 4$, let $R=\mathbb{Z} / 2$, and let $\mathbf{C}$ be a finite projective $R \Gamma_{G}$-complex which is an $R$-homology $\underline{n}$-sphere. If $1 \triangleleft K \triangleleft G$ with $K \cong \mathbb{Z} / 2$, then $\underline{n}(1)-\underline{n}(K)$ is even.

Proof. We consider the spectral sequence

$$
E_{2}^{s, t}=\operatorname{Ext}_{R \Gamma_{G}}^{s}\left(H_{t}(\mathbf{C}), M\right)
$$

with $M=I_{1} R$, which converges to $H^{s+t}\left(\operatorname{Hom}_{R \Gamma_{G}}(\mathbf{C}, M)\right)$. Write $n_{1}=\underline{n}(1), n_{K}=$ $\underline{n}(K)$, and $n_{G}=\underline{n}(G)$. Once again, the fact that $H^{k}(\mathbf{C} ; M)$ is zero in large dimensions $k>d=\operatorname{dim} \mathbf{C}(1)$ gives rise to a long exact sequence

$$
\begin{gathered}
\cdots \rightarrow \operatorname{Ext}_{R \Gamma_{G}}^{k+n_{1}-n_{G}+1}\left(I_{G} R, M\right) \stackrel{\delta}{\rightarrow} \operatorname{Ext}_{R \Gamma_{G}}^{k}\left(I_{1} R, M\right) \stackrel{\gamma}{\rightarrow} \operatorname{Ext}_{R \Gamma_{G}}^{k+n_{1}-n_{K}+1}\left(I_{K} R, M\right) \\
\rightarrow \operatorname{Ext}_{R \Gamma_{G}}^{k+n_{1}-n_{G}+2}\left(I_{G} R, M\right) \stackrel{\delta}{\rightarrow} \operatorname{Ext}_{R \Gamma_{G}}^{k+1}\left(I_{1} R, M\right) \rightarrow \cdots
\end{gathered}
$$

The analogue of Lemma 5.8 is easier in this case. We obtain

$$
\operatorname{Ext}_{R \Gamma_{G}}^{m}\left(I_{K} R, I_{1} R\right) \cong \operatorname{Ext}_{R \Gamma_{G}}^{m-1}\left(I_{1} R, I_{1} R\right) \cong H^{m-1}(G ; R)
$$

for every $m \geqslant 1$, and $\operatorname{Ext}_{R \Gamma_{G}}^{m}\left(I_{G} R, I_{1} R\right)=0$ for every $m \geqslant 0$. The vanishing result follows from the short exact sequence

$$
0 \rightarrow J_{K} R \rightarrow \underline{R} \rightarrow I_{G} R \rightarrow 0
$$

and the fact that $\operatorname{Ext}_{R \Gamma_{G}}^{m}\left(J_{K} R, I_{1} R\right)=0$, for $m \geqslant 0$, since $J_{K} R$ has a projective resolution consisting of modules of the form $E_{K} P$. On substituting these values into the long exact sequence, we obtain an isomorphism

$$
\gamma: H^{k}(G ; R) \cong H^{k+n_{1}-n_{K}}(G ; R)
$$

induced by cup product, for all large $k$. Since the cohomology ring $H^{*}(G ; R)$ modulo nilpotent elements is generated by a 2 -dimensional class, it follows that $n_{1}-n_{K}$ must be even.

Lemma 5.11 (Borel-Smith, part (iv)). Let $G=Q_{8}$, let $R=\mathbb{Z} / 2$, and let $\mathbf{C}$ be a finite projective $R \Gamma_{G}$-complex which is an R-homology $\underline{n}$-sphere. If $1 \triangleleft K \triangleleft G$ with $K \cong \mathbb{Z} / 2$, then $\underline{n}(1)-\underline{n}(K)$ is divisible by 4 .

Proof. This time we have three index 2 normal subgroups $H_{1}, H_{2}, H_{3}$, each isomorphic to $\mathbb{Z} / 4$. Write $n_{1}=\underline{n}(1), n_{K}=\underline{n}(K), n_{H_{i}}=\underline{n}\left(H_{i}\right)$, for $1 \leqslant i \leqslant 3$, and $n_{G}=$ $\underline{n}(G)$. We again consider the spectral sequence

$$
E_{2}^{s, t}=\operatorname{Ext}_{R \Gamma_{G}}^{s}\left(H_{t}(\mathbf{C}), M\right)
$$

with $M=I_{1} R$, which converges to $H^{s+t}\left(\operatorname{Hom}_{R \Gamma_{G}}(\mathbf{C}, M)\right)$. The exact sequences

$$
0 \rightarrow N \rightarrow \underline{R} \rightarrow I_{G} R \rightarrow 0
$$

and

$$
0 \rightarrow\left(J_{K} R\right)^{2} \rightarrow \oplus_{i} J_{H_{i}} R \rightarrow N \rightarrow 0
$$

lead to the calculation

$$
\operatorname{Ext}_{R \Gamma_{G}}^{m}\left(I_{G} R, I_{1} R\right)=0
$$


for every $m \geqslant 0$. The exact sequence

$$
0 \rightarrow I_{1} R \rightarrow J_{K} R \rightarrow I_{K} R \rightarrow 0
$$

implies that $\operatorname{Ext}_{R \Gamma_{G}}^{m}\left(I_{K} R, I_{1} R\right)=H^{m-1}(G ; R)$, for $m \geqslant 1$. Finally, the exact sequences

$$
0 \rightarrow J_{K} R \rightarrow J_{H_{i}} R \rightarrow I_{H_{i}} R \rightarrow 0
$$

show that $\operatorname{Ext}_{R \Gamma_{G}}^{m}\left(I_{H_{i}} R, I_{1} R\right)=0$, for $m \geqslant 0$ and $1 \leqslant i \leqslant 3$.

As a result of these calculations, we again obtain a 3 -line spectral sequence with corresponding long exact sequence

$$
\begin{gathered}
\cdots \rightarrow \operatorname{Ext}_{R \Gamma_{G}}^{k+n_{1}-n_{G}+1}\left(I_{G} R, M\right) \stackrel{\delta}{\rightarrow} \operatorname{Ext}_{R \Gamma_{G}}^{k}\left(I_{1} R, M\right) \stackrel{\gamma}{\rightarrow} \operatorname{Ext}_{R \Gamma_{G}}^{k+n_{1}-n_{K}+1}\left(I_{K} R, M\right) \\
\rightarrow \operatorname{Ext}_{R \Gamma_{G}}^{k+n_{1}-n_{G}+2}\left(I_{G} R, M\right) \stackrel{\delta}{\rightarrow} \operatorname{Ext}_{R \Gamma_{G}}^{k+1}\left(I_{1} R, M\right) \rightarrow \cdots
\end{gathered}
$$

in all large dimensions $k>d$. By the vanishing result above, the map

$$
\gamma: H^{k}(G ; R) \cong H^{k+n_{1}-n_{K}}(G ; R)
$$

is an isomorphism induced by cup product. Since the cohomology $\operatorname{ring} H^{*}(G ; R)$ modulo nilpotent elements is generated by a 4 -dimensional class, it follows that $n_{1}-$ $n_{K}$ is divisible by 4 .

Remark 5.12. The fact that the dimension function of an algebraic $\underline{n}$-homology sphere satisfies the Borel-Smith conditions suggests that more of the classical results on finite group actions on spheres might hold for finite projective chain complexes over a suitable orbit category. For example, one could ask for an algebraic version of the results of Dotzel-Hamrick [6] on $p$-groups. Other potential applications of algebraic models to finite group actions are outlined in $[7]$.

Example 5.13. An important test case for groups acting on spheres, or on products of spheres [1], is the rank 2 group $\operatorname{Qd}(p)=(\mathbb{Z} / p \times \mathbb{Z} / p) \rtimes S L_{2}(p)$. At present, it is not known whether $\mathrm{Qd}(p)$ can act freely on a product of two spheres, but Ünlü $[\mathbf{1 3}]$ showed that $\mathrm{Qd}(p)$ does not act on a finite complex homotopy equivalent to a sphere with rank 1 isotropy.

We can apply the Borel-Smith conditions prove an algebraic version of this result.

Proposition 5.14. Let $p$ be an odd prime, let $G=\mathrm{Qd}(p)$, let $R=\mathbb{Z} / p$, and let $\mathcal{F}$ be the family of all subgroups $H \leqslant G$ such that $\operatorname{rank}_{p}(H) \leqslant 1$. Let $\underline{n}$ be a super class function with $\underline{n}(1) \geqslant 0$. Then there exists no finite projective chain complex $\mathbf{C}$ over $R \Gamma_{G}$ which is an $R$-homology $\underline{n}$-sphere.

Proof. First observe that we can extend the family $\mathcal{F}$ to the family $\mathcal{S}(G)$ of all subgroups of $G$ by taking $\mathbf{C}(H)=0$ for all subgroups such that $H \notin \mathcal{F}$. Note that for these subgroups, we take $\underline{n}(H)=-1$. Observe that by Theorem $\mathrm{C}$ the dimension function $\underline{n}: \mathcal{S}(G) \rightarrow \mathbb{Z}$ satisfies the Borel-Smith conditions at the prime $p$.

Now the rest of the argument follows as in Ünlü [13, Theorem 3.3]. Let $P$ be a Sylow $p$-subgroup of $\mathrm{Qd}(p)$. The group $P$ is isomorphic to the extra-special $p$-group of order $p^{3}$ and exponent $p$. If $Z(P)$ is the center of $P$, then the quotient group $P / Z(P)$ is isomorphic to $\mathbb{Z} / p \times \mathbb{Z} / p$. Applying the Borel-Smith condition (ii) for this quotient, 
we get $\underline{n}(Z(P))=-1$. In $G$, it is possible to find two Sylow $p$-subgroups $P_{1}$ and $P_{2}$ such that $E=P_{1} \cap P_{2} \cong \mathbb{Z} / p \times \mathbb{Z} / p$ and $Z\left(P_{1}\right)$ and $Z\left(P_{2}\right)$ are distinct subgroups of order $p$ in $E$. Two such Sylow $p$-subgroups can be given as $P_{i}=(\mathbb{Z} / p \times \mathbb{Z} / p) \rtimes\left\langle A_{i}\right\rangle$ for $i=1,2$, where

$$
A_{1}=\left(\begin{array}{ll}
1 & 1 \\
0 & 1
\end{array}\right) \quad A_{2}=\left(\begin{array}{ll}
1 & 0 \\
1 & 1
\end{array}\right)
$$

Note that by the above argument $\underline{n}\left(Z\left(P_{i}\right)\right)=-1$, and non-central $p$-subgroups in $E$ are conjugate to each other. Therefore, we obtain that $\underline{n}(K)=-1$ for every subgroup $K$ of order $p$ in $E$. By the Borel-Smith conditions applied to $E$, we get $\underline{n}(1)=-1$, contradicting our assumption on $\underline{n}$.

\section{References}

[1] A. Adem and J. H. Smith, Periodic complexes and group actions, Ann. of Math. (2) 154 (2001), 407-435.

[2] D. J. Benson and J. F. Carlson, Projective resolutions and Poincaré duality complexes, Trans. Amer. Math. Soc. 342 (1994), 447-488.

[3] A. Borel, Seminar on transformation groups, With contributions by G. Bredon, E. E. Floyd, D. Montgomery, R. Palais. Annals of Mathematics Studies, No. 46, Princeton University Press, Princeton, N.J., 1960.

[4] K. S. Brown, Cohomology of Groups, Springer-Verlag, New York, 1994, Corrected reprint of the 1982 original.

[5] J. F. Carlson, L. Townsley, L. Valeri-Elizondo, and M. Zhang, Cohomology rings of finite groups, Algebras and Applications, vol. 3, Kluwer Academic Publishers, Dordrecht, 2003, With an appendix: Calculations of cohomology rings of groups of order dividing 64 by Carlson, Valeri-Elizondo and Zhang.

[6] R. M. Dotzel and G. C. Hamrick, p-Group actions on homology spheres, Invent. Math. 62 (1981), 437-442.

[7] J. Grodal and J. H. Smith, Algebraic models for finite G-spaces, Oberwolfach Reports, vol. 4, Eur. Math. Soc., Zürich, 2007, Abstracts from the Homotopy Theory workshop, September 16-22, 2007, organized by Paul Goerss, John Greenlees, and Stefan Schwede, pp. 2690-2692.

[8] I. Hambleton, S. Pamuk, and E. Yalçın, Equivariant $C W$-complexes and the orbit category, Comment. Math. Helv. 88 (2013), 369-425.

[9] I. Hambleton and E. Yalçı, Group actions on spheres with rank one isotropy, preprint (arXiv:1302.0507).

[10] W. Lück, Transformation groups and algebraic $K$-theory, Lecture Notes in Mathematics, vol. 1408, Springer-Verlag, Berlin, 1989, Mathematica Gottingensis.

[11] S. Pamuk, Periodic resolutions and finite group actions, Ph.D. thesis, McMaster University, 2008.

[12] T. tom Dieck, Transformation Groups, de Gruyter Studies in Mathematics, vol. 8, Walter de Gruyter \& Co., Berlin, 1987. 
[13] Ö. Ünlü, Constructions of free group actions on products of spheres, Ph.D. thesis, University of Wisconsin, 2004.

Ian Hambleton hambleton@mcmaster.ca

Department of Mathematics \& Statistics, McMaster University, Hamilton, Ontario L8S 4K1, Canada

Ergün Yalçın yalcine@fen.bilkent.edu.tr

Department of Mathematics, Bilkent University, 06800 Bilkent, Ankara, Turkey 